\title{
Study of a mathematical model for stimulated Raman scattering
}

\author{
T. BOUCHERES, T. COLIN, B. NKONGA, B. TEXIER ${ }^{1}$
}

Mathématiques appliqués de Bordeaux, Université Bordeaux 1 and CNRS

UMR 5466, 351 cours de la Libération, 33405 Talence cedex, France

A. BOURGEADE ${ }^{2}$

CEA-CESTA, BP2, 33114 Le Barp, France, LRC CEA MO3

\begin{abstract}
We study a semiclassical modelization of the interaction of a laser with a mono-atomic gas. The Maxwell equations are coupled with a three-level version of the Bloch equations. Taking into account the specificities of the laser pulse and of the gas, we introduce small parameters and a dimensionless form of the equations. To describe stimulated Raman scattering, we perform a three-scale WKB expansion in the weakly nonlinear regime of geometric optics. The limit system is of Schrödinger-Bloch type. We prove a global existence result for this system and the convergence of its solution toward the solution of the initial Maxwell-Bloch equations, as the parameter of the WKB expansion goes to 0 . We put in evidence Raman instability in the one-dimensional case, both theoretically and numerically.
\end{abstract}

\section{Physical context}

\subsection{Description of the model}

We consider the interaction of a laser pulse with a mono-atomic gas. As usual, the laser is described by the Maxwell equations [4]', [15]. The matter is described by the quantic Schrödinger equation. The link between these equations is made by the introduction of the polarization. The system is

$$
\left\{\begin{array}{l}
\partial_{t} \vec{B}+\operatorname{rot} \vec{E}=0, \\
\partial_{t} \vec{E}-c^{2} \operatorname{rot} \vec{B}=-\mu_{0} c^{2} \partial_{t} \vec{P}, \\
i \hbar \partial_{t} \boldsymbol{\Psi}=H \boldsymbol{\Psi}
\end{array}\right.
$$

where $\vec{E}, \vec{B}, \vec{P}$ designate the electric field, the magnetic field, and the (macroscopic) polarization. $\mu_{0}$ and $c$ denote respectively the permeability of free space and the velocity of light, $\hbar$ is the Planck constant. $\boldsymbol{\Psi}$ is the wave-function of the matter and $H$ its Hamiltonian. We suppose that the laser pulse propagates in a dilute atomic vapor. Hence, as a first approximation, one can neglect the coupling between the different atoms and take $\boldsymbol{\Psi}$ as the wave-function of one

\footnotetext{
${ }^{1}$ bouchere@math.u-bordeaux.fr, colin@math.u-bordeaux.fr, nkonga@math.u-bordeaux.fr, btexier@math.u-bordeaux.fr

2 bourgeade@cea.fr
} 
atom. We have $\vec{P}=N \vec{P}_{\text {atom }}$ where $N$ denotes the density of the gas and where $\vec{P}_{\text {atom }}$ is the microscopic polarization. As usual, the Hamiltonian $H$ is decomposed into $H=H_{0}+V$ where $H_{0}$ is the free Hamiltonian of the atom and $V$ the perturbation induced by the interaction with the electromagnetic field. We assume that the spectral decomposition of $H_{0}$ involves three eigenstates (denoted by $|0>| 1>,, \mid 1^{\prime}>$ ) associated with three eigenvalues which are not degenerate. The wave-function takes the form

$$
\boldsymbol{\Psi}(t)=a_{0}(t)\left|0>+a_{1}(t)\right| 1>+a_{1^{\prime}}(t) \mid 1^{\prime}>,
$$

and, associating $\mid 0>$ with the atomic ground state, the free Hamiltonian takes the form

$$
\mathbf{H}_{\mathbf{0}}=\left(\begin{array}{ccc}
0 & 0 & 0 \\
0 & \omega_{1} & 0 \\
0 & 0 & \omega_{1^{\prime}}
\end{array}\right) .
$$

Here the eigenvalue $\hbar \omega_{i}$ is the energy of the level i and $\omega_{i}$ the frequency associated with this level. For the perturbing part $V$ of the Hamiltonian, we restrict to the dipolar approximation $V=-e \vec{E} \cdot \vec{r}$, where $\vec{r}$ is the position operator.

With the notation $\rho_{i j}=a_{i} a_{j}^{*}$ and $\vec{p}_{j i}=e \int \vec{r}|i>| j>^{*} d \vec{r}$, the microscopic polarization is defined as

$$
\vec{P}_{\text {atom }}=\sum_{i, j} \rho_{i j} \vec{p}_{j i}=\operatorname{Tra}(\rho \vec{p}) .
$$

For simplicity, one can assume that the electromagnetic field is in transverse magnetic mode

$$
\vec{B}(t, x, y, z)=\left(\begin{array}{c}
B_{1}(t, x, y) \\
B_{2}(t, x, y) \\
0
\end{array}\right) \quad, \quad \vec{E}(t, x, y, z)=\left(\begin{array}{c}
0 \\
0 \\
E(t, x, y)
\end{array}\right) .
$$

We assume also that the electronic polarizations $\vec{p}_{i j}$ are singly oriented in the direction $\vec{e}_{z}=(0,0,1)$. Therefore, one has $\vec{P}=N\left(\sum_{i, j} a_{i} a_{j}^{*} p_{j i}\right) \vec{e}_{z}$. Clearly $p_{i j}=p_{j i}^{*}$ and we can choose the eigenstates in order to have $p_{i i}=0$. Moreover, by parity of energy levels, one of the $p_{i j}$ must vanish. In view of the Raman effect, the natural candidate is $p_{01^{\prime}}$ (see figure 1.1). This allows us to see that the polarizations $p_{01}, p_{11^{\prime}}$ can be taken real (to check this, take $\alpha=|\alpha| e^{i \psi} ; \gamma=$ $|\gamma| e^{i \varphi} ; \tilde{a_{0}}=a_{0} e^{-i \psi} ; \tilde{a_{1^{\prime}}}=a_{1^{\prime}} e^{i \varphi}$ and write the new system).

These assumptions lead to the following system:

$$
(\mathbf{P})\left\{\begin{array}{l}
\partial_{t} B_{1}+\partial_{y} E=0, \\
\partial_{t} B_{2}-\partial_{x} E=0, \\
\partial_{t} E-c^{2}\left(\partial_{x} B_{2}-\partial_{y} B_{1}\right)=-\mu_{0} c^{2} N \partial_{t}\left(\alpha a_{1} a_{0}^{*}+\gamma a_{1}^{*} a_{1^{\prime}}+\text { c.c }\right), \\
\partial_{t} a_{0}=i \frac{\alpha}{\hbar} E a_{1} \\
\partial_{t} a_{1}=-i \omega_{1} a_{1}+\frac{i}{\hbar} E\left(\alpha a_{0}+\gamma a_{1^{\prime}}\right), \\
\partial_{t} a_{1^{\prime}}=-i \omega_{1^{\prime}} a_{1^{\prime}}+i \frac{\gamma}{\hbar} E a_{1},
\end{array}\right.
$$


where $\alpha=p_{01}$ and $\gamma=p_{11^{\prime}}$.

In view of the stimulated Raman scattering, we want to study the evolution of a laser pulse $e^{i\left(\frac{k_{L} x-\omega_{L} t}{\epsilon}\right)} E_{L}+e^{i\left(\frac{k_{S} x-\omega_{S} t}{\epsilon}\right)} E_{S}$ with $E_{S} \ll E_{L}$ and where the frequencies $\omega_{L}, \omega_{S}$ are adjustable by an experimenter. One can wonder if this evolution could generate a growth of the $E_{S}$ component of the electric field. Such a behavior is actually possible. It has been discovered for the first time near 1923 and led to a great number of mathematical and physical studies. These studies have shown that this nonlinear effect is possible only if $\omega_{L}$ is close to the higher energy level $\omega_{1}$ and if $\omega_{S}$ (called the Raman frequency) satisfies the fundamental resonance assumption:

$$
\omega_{L}-\omega_{S} \approx \omega_{1^{\prime}}
$$

The situation is illustrated by the following figure:

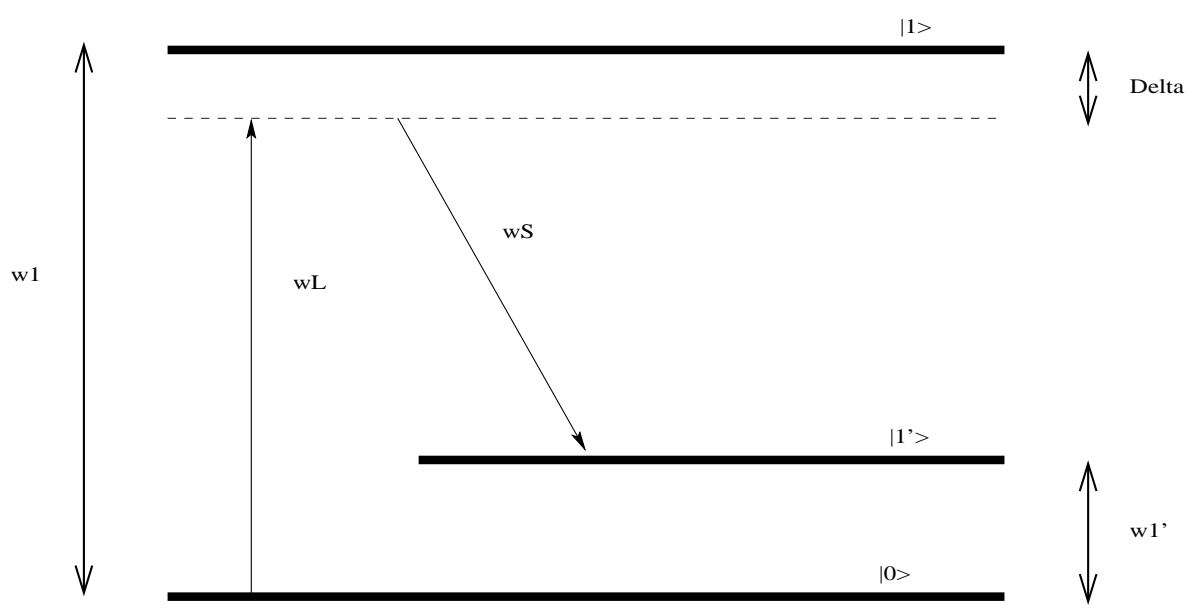

Figure 1.1: The three-level atom.

Let us discuss the limits of this model. First, it is clear that the consideration of 2D geometry (the TM mode for us) is a poor approximation of various physical states. In fact, when the general 3D geometry is considered, it is not realistic to assume that the electronic polarizations are singly oriented. Vectorial polarizations induce an important difference with the TM mode since in this case, $\partial_{t} \vec{P}=\left(\partial_{t} P\right) \vec{e}_{z}=F\left(a_{0} ; a_{1} ; a_{1^{\prime}}\right) \vec{e}_{z}$ whereas in the $3 \mathrm{D}$ case, we have $\partial_{t} \vec{P}=\widetilde{F}\left(a_{0} ; a_{1} ; a_{1^{\prime}} ; \vec{E}\right.$ ) (see also section 3.2). However, our theory applies in the general case, i.e without making the hypothesis that we are in the transverse magnetic mode. Concerning the dipolar approximation, it is widely used in the context of weakly nonlinear optics, i.e when the laser is not sufficiently intense to damage the matter (ionization or creation of a plasma for example). A more important problem is the treatment of the matter. The assumption of three non degenerate levels and of uncoupled atoms put aside many physical processes (among which the speed of atoms, the collisions, the thermodynamic fluctuations). In many modelizations, these processes are taken into account 
by a relaxation matrix which is added to the perturbing part of the Hamiltonian. Nevertheless, the main qualitative behavior of Raman scattering, that is the growth of the Stokes component of the electric field, appears in our simple model. This paper can be seen as a first step for the study of a kinetic-type model of the laser-matter interaction that would introduce the Doppler and Zeeman effects (i.e speed of atoms and degeneracy of energy levels). We present here a mathematical study of the basic model[4]' [15]. For more detailed models, see Bidegaray[2].

This paper is organized as follows.

In section 1.2, we introduce a dimensionless form of the system $(\mathbf{P})$ using the physical scale and we use the Bloch variables in order to obtain the system we deal with. In section 2, a WKB-type expansion is performed in order to obtain the Schrödinger-Bloch system which describes the Raman amplification. We give a rigorous error estimate (theorem 1). This result justifies SchrödingerBloch systems from Maxwell-Bloch systems. This a new result since a geometrical optics regime gives Schrödinger-like equations which is unusual (but see also Dumas[10]). In section 3, we state some global existence results for our systems (for the Maxwell-Bloch system, it is a consequence of work of Joly-MétivierRauch[12] and Dumas[9], for the Schrödinger-Bloch system, the result is new). In section 4 , we prove a instability result which is a rigorous result concerning Raman amplification in the $1 \mathrm{D}$ context. The last section is concerned with some numerical results illustrating the above results, especially the Raman growth.

\subsection{The physical scales and a dimensionless form of the equations}

The qualitative behaviors of system $(\mathbf{P})$ are intimately connected with the size of the parameters. Therefore, it is important to specify which physical applications we want to address. Here, we basically follow the adimensionalization process used by Colin-Nkonga [6].

We consider a dilute atomic gas with density $N \sim 5.10^{18}$ (to compare with the Avogadro number: $10^{24}$ ). The dipole coupling coefficients $\alpha$ and $\gamma$ are of size $|\alpha| \sim|\gamma| \sim 5.10^{-31}$. The frequencies $\omega_{i}$ associated with atomic states are usually closed to $10^{15}$. The frequencies $\omega_{L}$ and $\omega_{S}$ of the electric field have the same size and, for Raman scattering, we must have $\omega_{1^{\prime}}=\omega_{L}-\omega_{S} \sim 10^{15}$ and $\omega_{L}-\omega_{1}=\Delta \sim 10^{10}$ (note that $\Delta$ correspond to a wavelength $\lambda=2 \pi c / \Delta \sim 0.2 \mathrm{~m}$ which is not a laser wavelength). The fact that $\Delta \ll \omega_{1}$ means that our laser is nearly resonant with the matter: the interaction will exist but alone this process - i.e the transition from $\mid 0>$ to $\mid 1>-$ will be weak $[6]$. However, the assumption $\omega_{1^{\prime}}=\omega_{L}-\omega_{S}$ means that there will be moreover an exact resonance and this implies Raman scattering, i.e a strong transition from $\mid 1>$ to $\mid 1^{\prime}>$ and so between $\mid 0>$ and $\mid 1^{\prime}>$.

We consider a laser pulse which propagates along the x-axis. The duration of the pulse is $t_{0} \sim 2.10^{-8} \mathrm{~s}$. Characteristic lengths of propagation are $x_{0}=c t_{0} \sim 6 \mathrm{~m}$ 
and $y_{0} \sim 0.1 \mathrm{~m}$ for the transverse direction.

In order to have a dimensionless system, we set:

$$
E=E_{0} \widetilde{E}, B=B_{0} \widetilde{B}, t=t_{0} \widetilde{t}, x=x_{0} \widetilde{x}, y=y_{0} \widetilde{y}, \alpha=\mu \widetilde{\alpha}, \gamma=\mu \widetilde{\gamma} .
$$

The system is now (for simplicity the tilde are removed from the dimensionless variables):

$\left(\mathbf{P}^{\prime}\right)\left\{\begin{array}{l}\partial_{t} B_{1}+\frac{E_{0} t_{0}}{B_{0} y_{0}} \partial_{y} E=0, \\ \partial_{t} B_{2}-\frac{E_{0} t_{0}}{B_{0} x_{0}} \partial_{x} E=0, \\ \partial_{t} E-c^{2}\left(\frac{B_{0} t_{0}}{E_{0} x_{0}} \partial_{x} B_{2}-\frac{B_{0} t_{0}}{E_{0} y_{0}} \partial_{y} B_{1}\right)=-\mu_{0} c^{2} \mu \frac{N}{E_{0}} \partial_{t}\left(\alpha a_{1} a_{0}^{*}+\gamma a_{1}^{*} a_{1^{\prime}}+c . c\right), \\ \partial_{t} a_{0}=i \frac{\mu}{\hbar} \alpha E_{0} t_{0} E a_{1}, \\ \partial_{t} a_{1}=-i\left(\omega_{L}+\Delta\right) t_{0} a_{1}+i \frac{\mu}{\hbar} E_{0} t_{0} E\left(\alpha a_{0}+\gamma a_{1^{\prime}}\right), \\ \partial_{t} a_{1^{\prime}}=-i\left(\omega_{L}-\omega_{S}\right) t_{0} a_{1^{\prime}}+i \frac{\mu}{\hbar} \gamma E_{0} t_{0} E a_{1},\end{array}\right.$

The characteristic $E_{0}$ optical field is taken equal to the maximum amplitude of the incident pulse and, in order to have a unit speed in the x-axis, we take $\frac{E_{0} t_{0}}{B_{0} x_{0}}=1$.

Now, we define three important parameters:

$$
\epsilon=1 /\left(\omega_{L} t_{0}\right), \quad \eta=1 /\left(\Delta t_{0}\right), \quad \epsilon_{1}=y_{0} / x_{0},
$$

and we introduce the classical (time normalized) Rabi frequency $\omega_{r}=\frac{E_{0} \mu t_{0}}{\hbar}$. $\epsilon$ is the ratio of the duration of one period of light to the length of the pulse[6]. The size of $\epsilon$ is a quantification of how the time-envelope approximation will be efficient. $\eta$ measures the way the laser is nearly resonant with the transition from $\mid 0>$ to $\mid 1>$. $\epsilon_{1}$ has a geometrical meaning: it is related to the shape of the laser (it is the transverse size divided by the longitudinal ones; it is a small number).

For our physical scales, one has $\epsilon=1 / \omega_{L} t_{0} \sim 5.10^{-8}, \eta=1 / \Delta t_{0} \sim 5.10^{-3}$, and $\epsilon_{1}=y_{0} / x_{0} \sim 2.10^{-2}$.

Besides $\omega_{L} \sim 10^{15}, \omega_{s} \sim 10^{15}$ and $\omega_{L}-\omega_{s} \sim 10^{15}$, so that we must have $\omega_{s} t_{0}=\frac{b}{\epsilon}$ with $b \sim O(1) \neq 1$.

Finally, the coefficient of the polarization is evaluated by:

$$
\frac{\mu_{0} c^{2} N \mu}{E_{0}}=\frac{N \mu}{\epsilon_{0} E_{0}}=\frac{N \mu^{2} t_{0}}{\epsilon_{0} \omega_{r} \hbar} \sim 125.10^{-8}=g \epsilon \quad \text { with } \quad g \sim 25 .
$$

The resulting dimensionless system is

$$
\left(\mathbf{P}^{\prime \prime}\right)\left\{\begin{array}{l}
\partial_{t} B_{1}+\frac{1}{\epsilon_{1}} \partial_{y} E=0 \\
\partial_{t} B_{2}-\partial_{x} E=0 \\
\partial_{t} E-\left(\partial_{x} B_{2}-\frac{1}{\epsilon_{1}} \partial_{y} B_{1}\right)=-g \epsilon \partial_{t}\left(\alpha a_{1} a_{0}^{*}+\gamma a_{1}^{*} a_{1^{\prime}}+\text { c.c }\right), \\
\partial_{t} a_{0}=i \omega_{r} \alpha E a_{1} \\
\partial_{t} a_{1}=-i\left(\frac{1}{\epsilon}+\frac{1}{\eta}\right) a_{1}+i \omega_{r} E\left(\alpha a_{0}+\gamma a_{1^{\prime}}\right) \\
\partial_{t} a_{1^{\prime}}=-i\left(\frac{1-b}{\epsilon}\right) a_{1^{\prime}}+i \omega_{r} \gamma E a_{1}
\end{array}\right.
$$


Now we introduce a new set of quantic variables, the Bloch variables:

$Q_{1}=a_{0}^{*} a_{1} ; Q_{2}=a_{0}^{*} a_{1^{\prime}} ; Q_{3}=a_{1}^{*} a_{1^{\prime}} ; N_{1}=1-\left|a_{0}\right|^{2}+\left|a_{1}\right|^{2} ; N_{2}=\left|a_{1}\right|^{2}-\left|a_{1^{\prime}}\right|^{2}$.

The variables $Q_{i}$ measure the coherence between the different eigenstates. From this point of view, our model follows the classical statistical density matrix approach [4]' [15] for which the Schrödinger equation $i \hbar \partial_{t} \boldsymbol{\Psi}=H \boldsymbol{\Psi}$ is replaced by the Heisenberg equation of motion $i \hbar \partial_{t} \rho=[H, \rho]$.

The system is now:

$(\widetilde{\mathbf{P}})\left\{\begin{array}{l}\partial_{t} B_{1}+\frac{1}{\epsilon_{1}} \partial_{y} E=0, \\ \partial_{t} B_{2}-\partial_{x} E=0, \\ \partial_{t} E-\left(\partial_{x} B_{2}-\frac{1}{\epsilon_{1}} \partial_{y} B_{1}\right)=-g\left(-i \alpha Q_{1}-i \alpha \frac{\epsilon}{\eta} Q_{1}+i \gamma \frac{\epsilon}{\eta} Q_{3}+i b \gamma Q_{3}+\text { c.c }\right), \\ \partial_{t} Q_{1}=-i\left(\frac{1}{\epsilon}+\frac{1}{\eta}\right) Q_{1}+i \omega_{r} \gamma E Q_{2}+i \omega_{r} \alpha E\left(1-N_{1}\right), \\ \partial_{t} Q_{2}=-i\left(\frac{1-b}{\epsilon}\right) Q_{2}-i \omega_{r} \alpha E Q_{3}+i \omega_{r} \gamma E Q_{1}, \\ \partial_{t} Q_{3}=\left[i\left(\frac{1}{\epsilon}+\frac{1}{\eta}\right)-i\left(\frac{1-b}{\epsilon}\right)\right] Q_{3}+i \omega_{r} \gamma E N_{2}-i \omega_{r} \alpha E Q_{2}, \\ \partial_{t} N_{1}=4 \omega_{r} \alpha \operatorname{Im}\left(Q_{1}\right) E-2 \omega_{r} \gamma \operatorname{Im}\left(Q_{3}\right) E, \\ \partial_{t} N_{2}=2 \omega_{r} \alpha \operatorname{Im}\left(Q_{1}\right) E-4 \omega_{r} \gamma \operatorname{Im}\left(Q_{3}\right) E .\end{array}\right.$

One can note that the variables $\left(Q_{i}, N_{j}\right)$ are in one-to-one correspondence with $\left|a_{i}\right|^{2}$ (but not with $\left(a_{i}\right)$ ). So, for the fundamental conservation law of the system $\left(\mathbf{P}^{\prime \prime}\right)$

$$
\sum\left|a_{i}(t)\right|^{2}=\sum\left|a_{i}(0)\right|^{2}
$$

and with this one-to-one correspondence, one deduces the following conservation law for $(\widetilde{\mathbf{P}})$ :

$\sum\left(\left|Q_{i}(t)\right|^{2}-\left|Q_{i}(0)\right|^{2}\right)=\left(N_{1}(t) N_{2}(t)-N_{1}^{2}(t)-N_{2}^{2}(t)+N_{1}^{2}(0)+N_{2}^{2}(0)-N_{1}(0) N_{2}(0)\right) / 3$.

It is also important to note that the introduction of quantities $N_{i}$ is absolutely necessary for the study of Raman effect. Indeed, setting $C_{0}=\sum\left|a_{i}(0)\right|^{2}$, we have

$$
\left\{\begin{array}{l}
\left|a_{0}\right|^{2}=\left(C_{0} \pm \sqrt{C_{0}-4\left(\left|Q_{1}\right|^{2}+\left|Q_{2}\right|^{2}\right)}\right) / 2, \\
\left|a_{1}\right|^{2}=\left(C_{0} \pm \sqrt{C_{0}-4\left(\left|Q_{1}\right|^{2}+\left|Q_{3}\right|^{2}\right)}\right) / 2 \\
\left|a_{1^{\prime}}\right|^{2}=\left(C_{0} \pm \sqrt{C_{0}-4\left(\left|Q_{2}\right|^{2}+\left|Q_{3}\right|^{2}\right)}\right) / 2
\end{array}\right.
$$

and so we could, by the choice of a sign, express the variables $\left(Q_{3}, N_{1}, N_{2}\right)$ in term of $\left(Q_{1}, Q_{2}\right)$. For the sign $(+)$, this representation would be valid as long as $\left|a_{0}\right|^{2} \geq 1 / 2$. This approach has been used by Colin-Nkonga[6] for a two-level model but cannot be efficient for our problem because the growth of the Raman component of the electric field leads to inversion population (i.e $\left|a_{0}\right|^{2}<1 / 2$ ) even though the laser pulse is only quasi-resonant.

It still remains to introduce a nondimensional form of the quantic variables. As the laser is only almost resonant, it is natural to expect the quantic variables 
to remain small in a first time. Considering the equation on $Q_{1}$, expressing $N_{1}$ in term of $\left(Q_{1}, Q_{2}\right)$ as above (by the choice of the sign $(+)$ as we consider the atoms are in the ground state at time $t=0)$ and setting $Q_{1}=A \widetilde{Q_{1}}, Q_{2}=A \widetilde{Q_{2}}$ with $A \ll 1$, at first order in $A$, we have:

$$
\partial_{t} Q_{1}=-i\left(\frac{1}{\epsilon}+\frac{1}{\eta}\right) Q_{1}+i \omega_{r} \gamma E Q_{2}+i \omega_{r} \alpha E / A
$$

and integrating by parts leads to

$$
\begin{aligned}
Q_{1}(t)= & \int_{0}^{t} e^{-i(1 / \epsilon+1 / \eta)(t-s)}\left(i \omega_{r} \gamma E(s) Q_{2}(s)+i \omega_{r} \alpha E(s) / A\right) d s \\
= & {\left[\frac{e^{-i(1 / \epsilon+1 / \eta)(t-s)}}{(1 / \epsilon+1 / \eta)}\left(\omega_{r} \gamma E(s) Q_{2}(s)+\omega_{r} \alpha \frac{E(s)}{A}\right)\right]_{0}^{t} } \\
& +\int_{0}^{t} \frac{e^{-i(1 / \epsilon+1 / \eta)(t-s)}}{(1 / \epsilon+1 / \eta)}\left(\omega_{R} \gamma \partial_{t}\left(E Q_{2}\right)(s)+\omega_{R} \alpha \frac{\partial_{t} E(s)}{A}\right) d s .
\end{aligned}
$$

In order to have a $O(1)$ effect of the field on $Q_{1}$, it is necessary to choose $A=\omega_{r} \eta$. The fundamental law $\sum\left|a_{i}(t)\right|^{2}=1$ leads to $Q_{3}=A^{2} \widetilde{Q_{3}}, N_{1,2}=A^{2} \widetilde{N_{1,2}}$.

\section{Remark 1:}

The assumption $Q_{2}=A \widetilde{Q_{2}}$ is not natural. In fact, we can think that the eigenstate $\mid 1>$ will only play an intermediate rule in the transfer of population from $\mid 0>$ to $\mid 1^{\prime}>$ and that if $\eta$ influences the speed of this transfer, it does not influence its quantitative aspect. Indeed, look after the quantic part of system $\left(\mathbf{P}^{\prime \prime}\right)$, suppress the phase $\epsilon$ (what will be justified in the next paragraph), suppose the electric field is constant and compute the linear resulting system for both the non-resonant and resonant $(\eta=+\infty)$ case.

Setting $a=\omega_{r} \alpha E$ and $b=\omega_{r} \gamma E$, this leads, for the resonant case, to

$$
\left\{\begin{array}{l}
a_{1}(t)=i a \sin \left(\sqrt{a^{2}+b^{2}} t\right) / \sqrt{a^{2}+b^{2}}, \\
a_{1^{\prime}}(t)=-a b\left(1-\cos \left(\sqrt{a^{2}+b^{2}} t\right)\right) /\left(a^{2}+b^{2}\right), \\
a_{0}(t)=1-a^{2}\left(1-\cos \left(\sqrt{a^{2}+b^{2}} t\right)\right) /\left(a^{2}+b^{2}\right),
\end{array}\right.
$$

and for the non-resonant case ( with $\Delta=\sqrt{1+4 \eta^{2}\left(a^{2}+b^{2}\right)}$ and $\lambda_{ \pm}=\frac{-i}{2 \eta}(1 \pm$ $\Delta))$

$$
\left\{\begin{array}{l}
a_{1}(t)=2 i a \eta e^{-i t / 2 \eta} \frac{\sin (\Delta t)}{\Delta}, \\
a_{1^{\prime}}(t)=-\frac{a b}{a^{2}+b^{2}}-2 \eta^{2} \frac{a b}{\Delta}\left(\frac{e^{\lambda t}}{1-\Delta}-\frac{e^{\lambda_{-}}}{1+\Delta}\right), \\
a_{0}(t)=1-\frac{a^{2}}{a^{2}+b^{2}}-2 \eta^{2} \frac{a b}{\Delta}\left(\frac{e^{\lambda+t}}{1-\Delta}-\frac{e^{\lambda}-t}{1+\Delta}\right) .
\end{array}\right.
$$

Therefore, one can see that if $\left|a_{1}\right|_{\max }$ depends on $\eta$, it is not the case for $\left|a_{0}\right|_{\text {min }}$ and $\left|a_{1^{\prime}}\right|_{\max }$ (but the time on which these extremum will be taken increases toward $\infty$ when $\eta \longrightarrow 0$ ). Hence, it could seem more natural to take 
$Q_{1,3}=\left(\omega_{r} \eta\right) Q_{1,3}$ and not to change $Q_{2}, N_{1}, N_{2}$. We do not stress the influence of $\eta$ any longer because it is mainly for numerical schemes that this consideration is fundamental (in order to avoid stiffness).

To simplify the notations, we set

$$
c_{1}=g|\alpha|^{2} \omega_{r} \eta ; \quad c_{2}=\alpha^{2} \omega_{r}^{2} \eta ; \quad c_{3}=g \gamma c_{2} ; \quad c_{4}=\omega_{r} \gamma
$$

The final system is then

$$
(\mathbf{S})\left\{\begin{array}{l}
\partial_{t} B_{1}+\frac{1}{\epsilon_{1}} \partial_{y} E=0 \\
\partial_{t} B_{2}-\partial_{x} E=0 \\
\partial_{t} E-\left(\partial_{x} B_{2}-\frac{1}{\epsilon_{1}} \partial_{y} B_{1}\right)=i c_{1}\left(1+\frac{\epsilon}{\eta}\right) Q_{1}-i c_{3}(\epsilon+b \eta) Q_{3}+\text { c.c } \\
\partial_{t} Q_{1}=-i\left(\frac{1}{\epsilon}+\frac{1}{\eta}\right) Q_{1}+\frac{i}{\eta} E+i c_{4} E Q_{2}-i c_{2} E N_{1} \\
\partial_{t} Q_{2}=-i\left(\frac{1-b}{\epsilon}\right) Q_{2}-i c_{2} E Q_{3}+i c_{4} E Q_{1} \\
\partial_{t} Q_{3}=i\left(\frac{b}{\epsilon}+\frac{1}{\eta}\right) Q_{3}-\frac{i}{\eta} E Q_{2}+i c_{4} E N_{2} \\
\partial_{t} N_{1}=\frac{4}{\eta} \operatorname{Im}\left(Q_{1}\right) E-2 c_{4} \operatorname{Im}\left(Q_{2}\right) E \\
\partial_{t} N_{2}=\frac{2}{\eta} \operatorname{Im}\left(Q_{1}\right) E-4 c_{4} \operatorname{Im}\left(Q_{2}\right) E .
\end{array}\right.
$$

\section{Asymptotic analysis}

In the previous paragraph, we scaled the physical equations and exhibited three small parameters $\epsilon, \epsilon_{1}, \eta$. Our goal is now to perform an asymptotic analysis of system $(\mathbf{S})$ and obtain a reduced system that will be discretized easily. The aim of such a construction is to avoid the numerical computation of the oscillating phases $e^{i \theta / \epsilon}$ of our system. The adequate mathematical tools are the so-called WKB expansions (see JMR and al. for further details[7],'[11]'[14]).

In order to take into account the transverse dimension, we assume that $\epsilon_{1}=\sqrt{\frac{\epsilon}{L}}$ with $L \sim \mathcal{O}(1)$ for our physical applications and we perform a WKB expansion in the regime $\eta=$ constant with respect to $\epsilon$. The consideration of three spaces scale in the WKB expansion leads to an asymptotic regime that can be seen like a compromise between geometrical and diffractive standard regimes. Indeed, denoting by $E_{L}$ the component of the electric field which oscillates at the frequency $\omega_{L}$ and by $E_{S}$ the component that oscillates at the Raman frequency, it is shown that the evolution of these envelopes is governed by two parts: a transport along the direction of propagation and a Schrödinger component on the transverse variable. These equations are closed by the two distinct "source" terms $Q_{1}$ (for $E_{L}$ ) and $Q_{3}$ (for $E_{S}$ ) which are connected with a conservative system of ordinary differential equations.

System (S) has the form

$$
\mathcal{L}\left(\partial_{t}, \partial_{x}, \frac{1}{\epsilon_{1}} \partial_{y}, \frac{1}{\epsilon}\right) u=\partial_{t} u+A_{1} \partial_{x} u+A_{2} \partial_{y} u+\frac{L_{0}}{\epsilon} u=f(\epsilon, \eta, u),
$$


where $u=\left(B_{1} ; B_{2} ; E ; Q_{1} ; Q_{2} ; Q_{3} ; N_{1} ; N_{2}\right)^{T}$ and $A_{j}, L_{0}$ are defined by

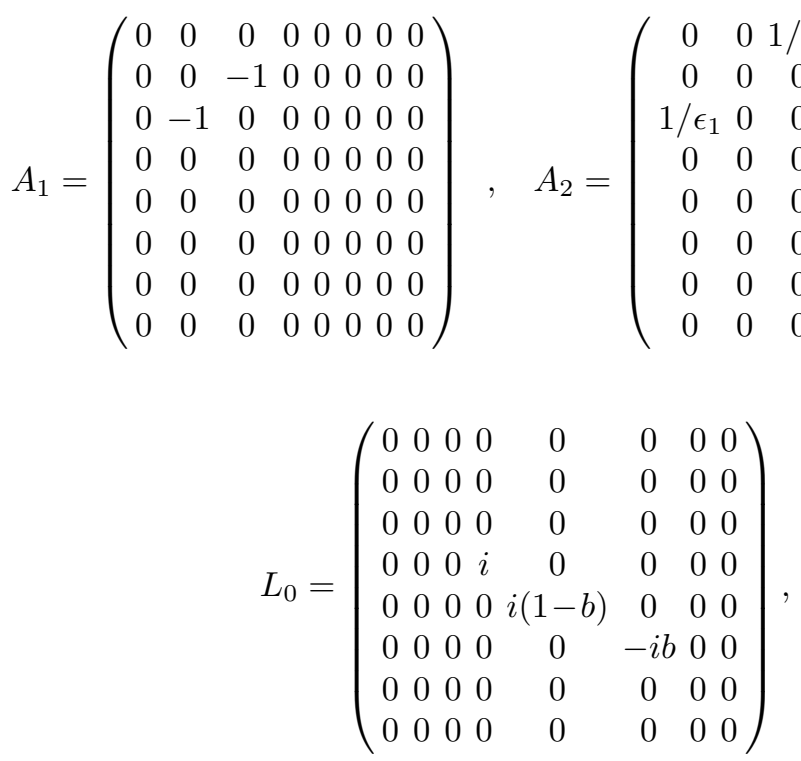

and $f(\epsilon, \eta, u)=L_{1}^{\epsilon, \eta} u+B^{\eta}(u, u)$ where $L_{1}^{\epsilon, \eta}$ is linear, $B^{\eta}$ is bilinear and both terms are bounded with respect to $\epsilon$.

As usual, we define the characteristic variety by

$$
\operatorname{Char}(\mathcal{L})=\left\{(\omega, k) \in \mathbb{R} \times \mathbb{R}^{2}, \operatorname{det}\left(-\omega I+\sum A_{i} k_{i}+L_{0} / i\right)=0\right\} .
$$

Setting $|k|_{\epsilon_{1}}=\sqrt{k_{1}^{2}+k_{2}^{2} / \epsilon_{1}^{2}}$, we have

$$
\operatorname{Char}(\mathcal{L})=\left\{(0 ; k),(1 ; k),(1-b ; k),(-b ; k),\left(|k|_{\epsilon_{1}} ; k\right),\left(|k|_{\epsilon_{1}} ; k\right) \quad / \forall k \in \mathbb{R}^{2}\right\} .
$$

This characteristic variety is pictured below in the 1D case (Figure 1.2).

Now, in view of stimulated Raman scattering, we are concerned with the propagation of a solution corresponding to a Cauchy data of the form $E(t=0)=$ $E_{L}(x, y) e^{i \frac{x}{\epsilon}}+E_{S}(x, y) e^{i b \frac{x}{\epsilon}}$. Hence, our WKB expansion must take into account the harmonics $e^{i \frac{x-t}{\epsilon}}$ and $e^{i b \frac{x-t}{\epsilon}}$. Geometrically, this means that we build our asymptotic solution on the singular points $(b ;-b)$ and $(1 ; 1)$ of $C h a r(\mathcal{L})$. In fact, using the terminology of Joly-Métivier-Rauch, we are in a geometrical optics regime, but we will obtain a Schrödinger-like model. This is mainly due to the presence of the transverse scale $1 / \sqrt{\epsilon}$. The proof however follows the classical ones: we first perform a formal asymptotic expansion, then we construct an approximate solution and we finally prove an error estimate using usual energy estimates. 


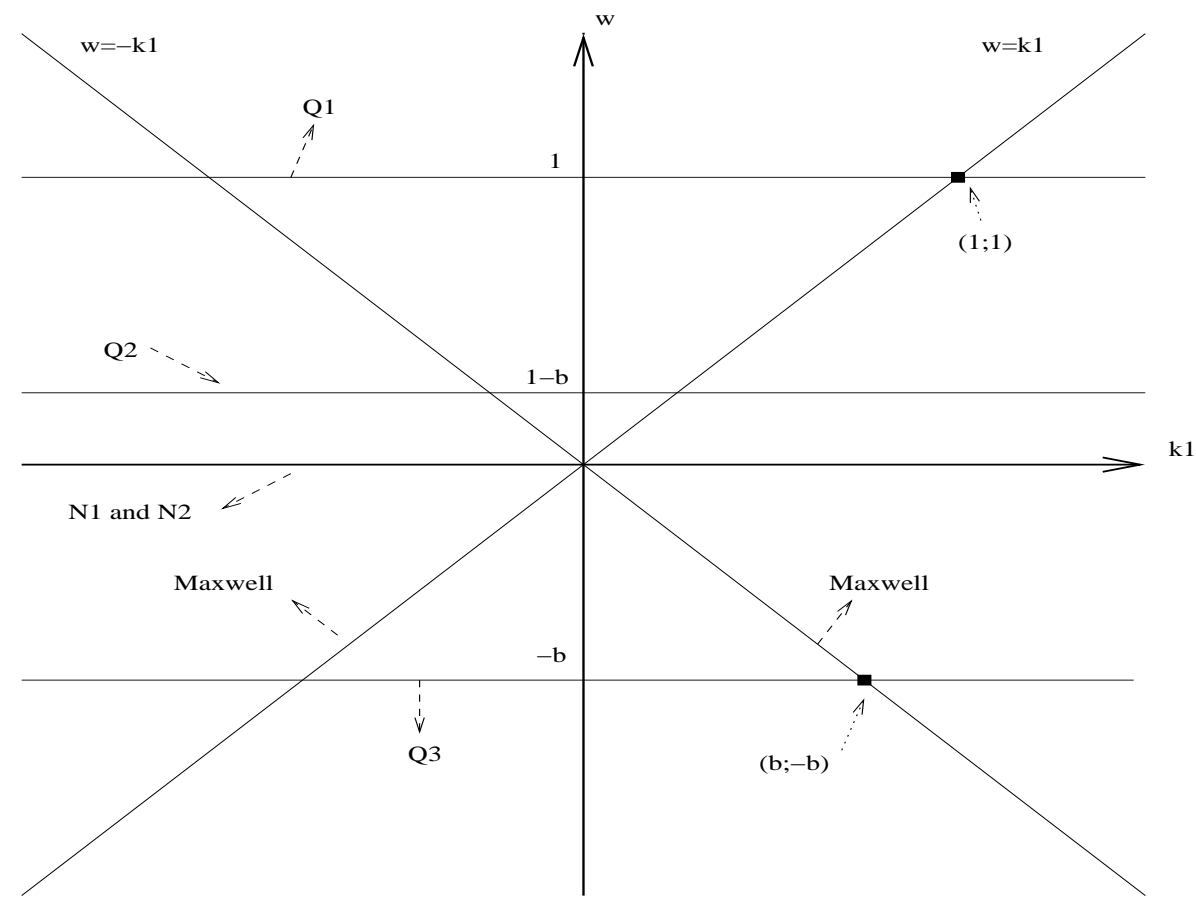

Figure 1.2: The characteristic variety.

\section{$2.1 \quad$ Formal asymptotic expansion}

The first step is to seek for a solution of $(\mathbf{S})$ in the form

$u^{\epsilon}(t, x, y)=\left(B_{1}, B_{2}, E, Q_{1}, Q_{2}, Q_{3}, N_{1}, N_{2}\right)(t, x, y)=\mathbf{u}\left(t, x, y, \theta_{1}, \theta_{2}\right)_{\left|\theta_{1}=\frac{x-t}{\epsilon},\right| \theta_{2}=b \frac{x-t}{\epsilon}}$,

where $\mathbf{u}$ is supposed to be $2 \pi$-periodic in $\theta_{1}$ and $\theta_{2}$. We assume the phases $\theta_{i}$ to be $\mathbb{Q}$-linearly independent (i.e $b \in \mathbb{R} / \mathbb{Q}$ ). Otherwise, one is reduced to the single phase case.

We impose $\mathbf{u}\left(t, x, y, \theta_{1}, \theta_{2}\right):=\left(\mathbf{B}_{\mathbf{1}}, \mathbf{B}_{\mathbf{2}}, \mathbf{E}, \mathbf{Q}_{\mathbf{1}}, \mathbf{Q}_{\mathbf{2}}, \mathbf{Q}_{\mathbf{3}}, \mathbf{N}_{\mathbf{1}}, \mathbf{N}_{\mathbf{2}}\right)\left(t, x, y, \theta_{1}, \theta_{2}\right)$ to 
satisfies the following singular equation:

- $\left(-\frac{1}{\epsilon} \partial_{\theta_{1}}-\frac{b}{\epsilon} \partial_{\theta_{2}}+\partial_{t}\right) \mathbf{B}_{2}-\left(\frac{1}{\epsilon} \partial_{\theta_{1}}+\frac{b}{\epsilon} \partial_{\theta_{2}}+\partial_{x}\right) \mathbf{E}=0$

- $\left(-\frac{1}{\epsilon} \partial_{\theta_{1}}-\frac{b}{\epsilon} \partial_{\theta_{2}}+\partial_{t}\right) \mathbf{B}_{1}+\frac{\sqrt{L}}{\sqrt{\epsilon}} \partial_{y} \mathbf{E}=0$,

- $\left(-\frac{1}{\epsilon} \partial_{\theta_{1}}-\frac{b}{\epsilon} \partial_{\theta_{2}}+\partial_{t}\right) \mathbf{E}-\left(\frac{1}{\epsilon} \partial_{\theta_{1}}+\frac{b}{\epsilon} \partial_{\theta_{2}}+\partial_{x}\right) \mathbf{B}_{\mathbf{2}}+\frac{\sqrt{L}}{\sqrt{\epsilon}} \partial_{y} \mathbf{B}_{\mathbf{1}}$

$$
=i c_{1}(\epsilon+\eta) \mathbf{Q}_{\mathbf{1}}-i c_{3}(\epsilon+b \eta) \mathbf{Q}_{\mathbf{3}}+c . c,
$$

- $\left(-\frac{1}{\epsilon} \partial_{\theta_{1}}-\frac{b}{\epsilon} \partial_{\theta_{2}}+\partial_{t}\right) \mathbf{Q}_{\mathbf{1}}=-i\left(\frac{1}{\epsilon}+\frac{1}{\eta}\right) \mathbf{Q}_{\mathbf{1}}+i \frac{\mathbf{E}}{\eta}+i c_{4} \mathbf{E Q}_{\mathbf{2}}+-i c_{2} \mathbf{E N}_{\mathbf{1}}$,

- $\left(-\frac{1}{\epsilon} \partial_{\theta_{1}}-\frac{b}{\epsilon} \partial_{\theta_{2}}+\partial_{t}\right) \mathbf{Q}_{\mathbf{2}}=-i \frac{1-b}{\epsilon} \mathbf{Q}_{\mathbf{2}}+i c_{4} \mathbf{E} \mathbf{Q}_{\mathbf{1}}-i c_{2} \mathbf{E} \mathbf{Q}_{\mathbf{3}}$,

- $\left(-\frac{1}{\epsilon} \partial_{\theta_{1}}-\frac{b}{\epsilon} \partial_{\theta_{2}}+\partial_{t}\right) \mathbf{Q}_{\mathbf{3}}=i\left(\frac{1}{\eta}+\frac{b}{\epsilon}\right) \mathbf{Q}_{\mathbf{3}}-i \frac{\mathbf{E} \mathbf{Q}_{\mathbf{2}}}{\eta}+i c_{4} \mathbf{E N}_{\mathbf{2}}$,

- $\left(-\frac{1}{\epsilon} \partial_{\theta_{1}}-\frac{b}{\epsilon} \partial_{\theta_{2}}+\partial_{t}\right) \mathbf{N}_{\mathbf{1}}=4 \frac{\mathbf{E} \operatorname{Im} \mathbf{Q}_{\mathbf{1}}}{\eta}-2 c_{4} \mathbf{E} \operatorname{Im} \mathbf{Q}_{\mathbf{3}}$,

- $\left(-\frac{1}{\epsilon} \partial_{\theta_{1}}-\frac{b}{\epsilon} \partial_{\theta_{2}}+\partial_{t}\right) \mathbf{N}_{\mathbf{2}}=2 \frac{\mathbf{E} \operatorname{Im} \mathbf{Q}_{\mathbf{1}}}{\eta}-4 c_{4} \mathbf{E} \operatorname{Im} \mathbf{Q}_{\mathbf{3}}$,

for all $\left(t, x, y, \theta_{1}, \theta_{2}\right) \in[0, T] \times \mathbb{R}^{2} \times \mathbb{T}^{2}$.

Now, setting

$$
\mathbf{u}=\mathbf{u}^{1}+\sqrt{\epsilon} \mathbf{u}^{2}+\epsilon \mathbf{u}^{3}+O\left(\epsilon^{3 / 2}\right),
$$

plugging into $(2.2)-(2.9)$ and collecting terms according to the powers of $\epsilon$ lead to:

\section{$\operatorname{Order} \mathcal{O}(1 / \epsilon)$}

- $-\left(\partial_{\theta_{1}}+b \partial_{\theta_{2}}\right) \mathbf{E}^{\mathbf{1}}-\left(\partial_{\theta_{1}}+b \partial_{\theta_{2}}\right) \mathbf{B}_{\mathbf{2}}^{\mathbf{1}}=0$,

- $-\left(\partial_{\theta_{1}}+b \partial_{\theta_{2}}\right) \mathbf{B}_{1}^{1}=0$,

- $-\left(\partial_{\theta_{1}}+b \partial_{\theta_{2}}\right) \mathbf{Q}_{1}^{\mathbf{1}}=-i \mathbf{Q}_{1}^{\mathbf{1}}$,

- $-\left(\partial_{\theta_{1}}+b \partial_{\theta_{2}}\right) \mathbf{Q}_{\mathbf{2}}^{\mathbf{1}}=-i(1-b) \mathbf{Q}_{\mathbf{2}}^{\mathbf{1}}$,

- $-\left(\partial_{\theta_{1}}+b \partial_{\theta_{2}}\right) \mathbf{Q}_{\mathbf{3}}^{\mathbf{1}}=i b \mathbf{Q}_{\mathbf{3}}^{\mathbf{1}}$,

- $-\left(\partial_{\theta_{1}}+b \partial_{\theta_{2}}\right) \mathbf{N}_{\mathbf{1}}^{\mathbf{1}}=0$,

- $-\left(\partial_{\theta_{1}}+b \partial_{\theta_{2}}\right) \mathbf{N}_{\mathbf{2}}^{\mathbf{1}}=0$, 
Order $\mathcal{O}(1 / \sqrt{\epsilon})$

$$
\begin{aligned}
& \text { - }-\left(\partial_{\theta_{1}}+b \partial_{\theta_{2}}\right) \mathbf{E}^{2}-\left(\partial_{\theta_{1}}+b \partial_{\theta_{2}}\right) \mathbf{B}_{2}^{2}+\sqrt{L} \partial_{y} \mathbf{B}_{1}^{1}=0, \\
& \text { - }-\left(\partial_{\theta_{1}}+b \partial_{\theta_{2}}\right) \mathbf{E}^{2}-\left(\partial_{\theta_{1}}+b \partial_{\theta_{2}}\right) \mathbf{B}_{2}^{2}=0, \\
& \text { - }-\left(\partial_{\theta_{1}}+b \partial_{\theta_{2}}\right) \mathbf{B}_{1}^{2}+\sqrt{L} \partial_{y} \mathbf{E}^{\mathbf{1}}=0, \\
& \text { - }-\left(\partial_{\theta_{1}}+b \partial_{\theta_{2}}\right) \mathbf{Q}_{1}^{2}=-i \mathbf{Q}_{1}^{2}, \\
& \text { - }-\left(\partial_{\theta_{1}}+b \partial_{\theta_{2}}\right) \mathbf{Q}_{2}^{2}=-i(1-b) \mathbf{Q}_{\mathbf{2}}^{2}, \\
& \text { - }-\left(\partial_{\theta_{1}}+b \partial_{\theta_{2}}\right) \mathbf{Q}_{3}^{2}=i b \mathbf{Q}_{\mathbf{3}}^{2}, \\
& \text { - }-\left(\partial_{\theta_{1}}+b \partial_{\theta_{2}}\right) \mathbf{N}_{\mathbf{1}}^{2}=0, \\
& \text { - }-\left(\partial_{\theta_{1}}+b \partial_{\theta_{2}}\right) \mathbf{N}_{\mathbf{2}}^{2}=0,
\end{aligned}
$$

Order $\mathcal{O}(1)$

$$
\begin{aligned}
& \text { - } \partial_{t} \mathbf{B}_{2}^{1}-\left(\partial_{\theta_{1}}+b \partial_{\theta_{2}}\right) \mathbf{B}_{2}^{\mathbf{3}}-\partial_{x} \mathbf{E}^{\mathbf{1}}-\left(\partial_{\theta_{1}}+b \partial_{\theta_{2}}\right) \mathbf{E}^{\mathbf{3}}=0 \text {, } \\
& \text { - } \partial_{t} \mathbf{B}_{1}^{1}-\left(\partial_{\theta_{1}}+b \partial_{\theta_{2}}\right) \mathbf{B}_{1}^{3}+\sqrt{L} \partial_{y} \mathbf{E}^{3}=0 \text {, } \\
& \text { - } \partial_{t} \mathbf{E}^{1}-\left(\partial_{\theta_{1}}+b \partial_{\theta_{2}}\right) \mathbf{E}^{3}-\partial_{x} \mathbf{B}_{2}^{1}+\sqrt{L} \partial_{y} \mathbf{B}_{1}^{2}-\left(\partial_{\theta_{1}}+b \partial_{\theta_{2}}\right) \mathbf{B}_{2}^{3} \\
& =-i b c_{3} \eta \mathbf{Q}_{\mathbf{3}}^{\mathbf{1}}+i c_{1} \mathbf{Q}_{\mathbf{1}}^{\mathbf{1}}+\text { c.c, } \\
& \text { - } \partial_{t} \mathbf{Q}_{\mathbf{1}}^{\mathbf{1}}-\left(\partial_{\theta_{1}}+b \partial_{\theta_{2}}\right) \mathbf{Q}_{\mathbf{1}}^{3}=-\frac{i}{\eta} \mathbf{Q}_{\mathbf{1}}^{\mathbf{1}}-i \mathbf{Q}_{\mathbf{1}}^{3}+i c_{4} \mathbf{E}^{\mathbf{1}} \mathbf{Q}_{\mathbf{2}}^{1}-i c_{2} \mathbf{E}^{\mathbf{1}} \mathbf{N}_{\mathbf{1}}^{\mathbf{1}} \text {, } \\
& \text { - } \partial_{t} \mathbf{Q}_{2}^{1}-\left(\partial_{\theta_{1}}+b \partial_{\theta_{2}}\right) \mathbf{Q}_{2}^{3}=-i(1-b) \mathbf{Q}_{2}^{3}+i c_{4} \mathbf{E}^{\mathbf{1}} \mathbf{Q}_{\mathbf{1}}^{\mathbf{1}}-i c_{2} \mathbf{E}^{\mathbf{1}} \mathbf{Q}_{\mathbf{3}}^{\mathbf{1}} \text {, } \\
& \text { - } \partial_{t} \mathbf{Q}_{\mathbf{3}}^{\mathbf{1}}-\left(\partial_{\theta_{1}}+b \partial_{\theta_{2}}\right) \mathbf{Q}_{\mathbf{3}}^{\mathbf{3}}=i b \mathbf{Q}_{\mathbf{3}}^{\mathbf{3}}+\frac{i}{\eta} \mathbf{Q}_{\mathbf{3}}^{\mathbf{1}}-\frac{i}{\eta} \mathbf{E}^{\mathbf{1}} \mathbf{Q}_{\mathbf{2}}^{\mathbf{1}}+i c_{4} \mathbf{E}^{\mathbf{1}} \mathbf{N}_{\mathbf{2}}^{\mathbf{1}} \text {, } \\
& \text { - } \partial_{t} \mathbf{N}_{\mathbf{1}}^{\mathbf{1}}-\left(\partial_{\theta_{1}}+b \partial_{\theta_{2}}\right) \mathbf{N}_{\mathbf{1}}^{\mathbf{3}}=\frac{4}{\eta} \mathbf{E}^{\mathbf{1}} \operatorname{Im} \mathbf{Q}_{\mathbf{1}}^{\mathbf{1}}-2 c_{4} \mathbf{E}^{\mathbf{1}} \operatorname{Im} \mathbf{Q}_{\mathbf{3}}^{\mathbf{1}} \text {, } \\
& \text { - } \partial_{t} \mathbf{N}_{\mathbf{2}}^{1}-\left(\partial_{\theta_{1}}+b \partial_{\theta_{2}}\right) \mathbf{N}_{\mathbf{2}}^{\mathbf{3}}=\frac{2}{\eta} \mathbf{E}^{\mathbf{1}} \operatorname{Im} \mathbf{Q}_{\mathbf{1}}^{1}-4 c_{4} \mathbf{E}^{\mathbf{1}} \operatorname{Im} \mathbf{Q}_{\mathbf{3}}^{\mathbf{1}} \text {. }
\end{aligned}
$$

The profiles $\mathbf{u}^{\mathbf{j}}$ are now expanded in Fourier series as:

$$
\mathbf{u}^{\mathbf{j}}\left(t, x, y, \theta_{1}, \theta_{2}\right)=\sum_{p_{i} \in \mathbb{Z}^{2}} u_{p_{1}, p_{2}}^{j}(t, x, y) e^{i\left(p_{1} \theta_{1}+p_{2} \theta_{2}\right)} .
$$

We obtain:

Order $\mathcal{O}(1 / \epsilon)$

- $(2.10) \Rightarrow\left(p_{1}+b p_{2}\right)\left(E_{p_{1}, p_{2}}^{1}+B_{2, p_{1}, p_{2}}^{1}\right)=0$,

- $(2.11) \Rightarrow\left(p_{1}+b p_{2}\right) B_{1, p_{1}, p_{2}}^{1}=0$,

- $(2.12) \Rightarrow\left(1-p_{1}-b p_{2}\right) Q_{1, p_{1}, p_{2}}^{1}=0$,

- $(2.13) \Rightarrow\left(1-b-p_{1}-b p_{2}\right) Q_{2, p_{1}, p_{2}}^{1}=0$,

- $(2.14) \Rightarrow\left(b+p_{1}+b p_{2}\right) Q_{3, p_{1}, p_{2}}^{1}=0$,

- $(2.15) \Rightarrow\left(p_{1}+b p_{2}\right) N_{1, p_{1}, p_{2}}^{1}=0$,

- $(2.16) \Rightarrow\left(p_{1}+b p_{2}\right) N_{2, p_{1}, p_{2}}^{1}=0$, 
Order $\mathcal{O}(1 / \sqrt{\epsilon})$

- $(2.20) \Rightarrow\left(1-p_{1}-b p_{2}\right) Q_{1, p_{1}, p_{2}}^{2}=0$,

- $(2.21) \Rightarrow\left(1-b-p_{1}-b p_{2}\right) Q_{2, p_{1}, p_{2}}^{2}=0$,

- $(2.19) \Rightarrow\left(p_{1}+b p_{2}\right) B_{1, p_{1}, p_{2}}^{2}+i \sqrt{L} \partial_{y} E_{p_{1}, p_{2}}^{1}=0$,

- $(2.18) \Rightarrow\left(p_{1}+b p_{2}\right)\left(B_{2, p_{1}, p_{2}}^{2}+E_{p_{1}, p_{2}}^{2}\right)=0$.

As $b$ is irrational, we have the following relations

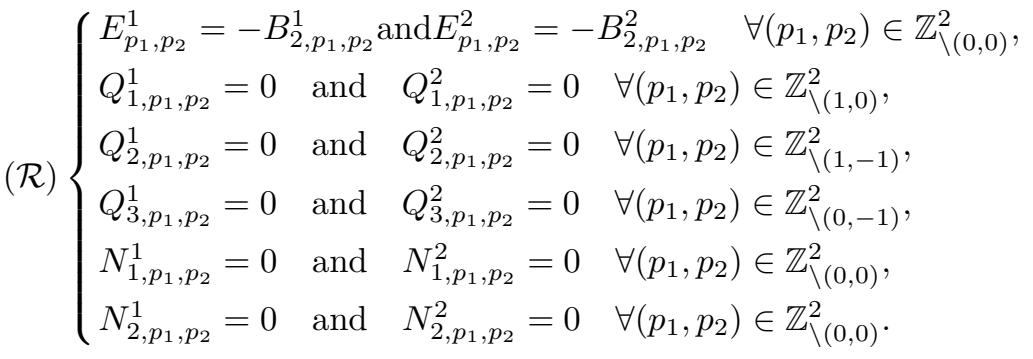

We plug (2.25) into (2.27) and obtain

$$
\left(\partial_{t}+\partial_{x}\right)\left(\mathbf{E}^{\mathbf{1}}-\mathbf{B}_{\mathbf{2}}^{\mathbf{1}}\right)+\sqrt{L} \partial_{y} \mathbf{B}_{\mathbf{1}}^{\mathbf{2}}=i c_{1} \mathbf{Q}_{\mathbf{1}}^{\mathbf{1}}-i c_{3} \eta b \mathbf{Q}_{\mathbf{3}}^{\mathbf{1}}+\text { c.c. }
$$

$(2.10)$ and $(2.19)$ gives, for $\left(p_{1} ; p_{2}\right) \neq(0 ; 0)$, the relation

$$
\left(2 \partial_{t}+2 \partial_{x}-i \frac{L}{\left(p_{1}+b p_{2}\right)} \partial_{y^{2}}^{2}\right) E_{p_{1}, p_{2}}^{1}=i c_{1} Q_{1, p_{1}, p_{2}}^{1}-i c_{3} \eta b Q_{3, p_{1}, p_{2}}^{1}+\text { c.c. }
$$

We have

$$
\left\{\begin{array}{c}
\left(p_{1}, p_{2}\right) \in \mathbb{Z}_{\backslash\{(1,0) ;(-1,0) ;(0,-1) ;(0,1) ;(0,0)\}}^{2} \\
\Rightarrow 2\left(\partial_{t}+\partial_{x}\right) E_{p_{1}, p_{2}}^{1}-i \frac{L}{\left(p_{1}+b p_{2}\right)} \partial_{y^{2}}^{2} E_{p_{1}, p_{2}}^{1}=0 .
\end{array}\right.
$$

Consequently, if $E_{p_{1}, p_{2}}^{1}(0)=0$ then $E_{p_{1}, p_{2}}^{1}(t)=0$.

For the other harmonics, we have

$$
\left\{\begin{array}{l}
2\left(\partial_{t}+\partial_{x}\right) E_{0,-1}^{1}+i \frac{L}{b} \partial_{y^{2}}^{2} E_{0,-1}^{1}=-i b c_{3} \eta Q_{3,0,-1}^{1} \\
2\left(\partial_{t}+\partial_{x}\right) E_{0,1}^{1}-i \frac{L}{b} \partial_{y^{2}}^{2} E_{0,1}^{1}=i b c_{3} \eta Q_{3,0,-1}^{1 *} \\
2\left(\partial_{t}+\partial_{x}\right) E_{1,0}^{1}-i L \partial_{y^{2}}^{2} E_{1,0}^{1}=i c_{1} Q_{1,1,0}^{1} \\
2\left(\partial_{t}+\partial_{x}\right) E_{-1,0}^{1}+i L \partial_{y^{2}}^{2} E_{-1,0}^{1}=-i c_{1} \eta Q_{1,1,0}^{1 *}
\end{array}\right.
$$

hence, by uniqueness, $E_{1,0}^{1}=E_{-1,0}^{1 *}$ and $E_{0,1}^{1}=E_{0,-1}^{1 *}$.

Similarly, the equations for the quantic variables are

$$
\left\{\begin{array}{l}
\partial_{t} Q_{1,1,0}^{1}=-\frac{i}{\eta} Q_{1,1,0}^{1}+\frac{i}{\eta} E_{1,0}^{1}-i c_{2} E_{1,0}^{1} N_{1,0,0}^{1}+i c_{4} Q_{2,1,-1}^{1} E_{0,1}^{1}, \\
\partial_{t} Q_{2,1,-1}^{1}=-i c_{2} E_{1,0}^{1} Q_{3,0,-1}^{1}+i c_{4} Q_{1,1,0}^{1} E_{0,1}^{1 *} \\
\partial_{t} Q_{3,0,-1}^{1}=\frac{i}{\eta} Q_{3,0,-1}^{1}-\frac{i}{\eta} E_{1,0}^{1 *} Q_{2,1,-1}^{1}+i c_{4} N_{2,0,0}^{1} E_{0,1}^{1 *}, \\
\partial_{t} N_{1,0,0}^{1}=-\frac{4}{\eta} \operatorname{Im}\left(E_{1,0}^{1} Q_{1,1,0}^{1 *}\right)-2 c_{4} \operatorname{Im}\left(E_{0,1}^{1} Q_{3,0,-1}^{1}\right) \\
\partial_{t} N_{2,0,0}^{1}=-\frac{2}{\eta} \operatorname{Im}\left(E_{1,0}^{1} Q_{1,1,0}^{1 *}\right)-4 c_{4} \operatorname{Im}\left(E_{0,1}^{1} Q_{3,0,-1}^{1}\right) .
\end{array}\right.
$$




\subsection{Construction of the approximate solution}

We are now able to construct an approximate solution to $(\mathbf{S})$. In this view, let us consider the following system:

$$
(\mathbf{M})\left\{\begin{array}{l}
\left(\partial_{t}+\partial_{x}\right) E_{S}-i \frac{L}{2 b} \partial_{y^{2}}^{2} E_{S}=i \frac{b}{2} c_{3} \eta Q_{3} \\
\left(\partial_{t}+\partial_{x}\right) E_{L}-i \frac{L}{2} \partial_{y^{2}}^{2} E_{L}=i \frac{c_{1}}{2} \eta Q_{1} \\
\partial_{t} Q_{1}=-\frac{i}{\eta} Q_{1}+\frac{i}{\eta} E_{L}+i c_{4} E_{S} Q_{2}-i c_{2} E_{L} N_{1} \\
\partial_{t} Q_{2}=-i c_{2} E_{L} Q_{3}^{*}+i c_{4} E_{S}^{*} Q_{1} \\
\partial_{t} Q_{3}=-\frac{i}{\eta} Q_{3}+\frac{i}{\eta} E_{L} Q_{2}^{*}-i c_{4} E_{S} N_{2} \\
\partial_{t} N_{1}=-\frac{4}{\eta} \operatorname{Im}\left(E_{L} Q_{1}^{*}\right)-2 c_{4} \operatorname{Im}\left(E_{S} Q_{3}^{*}\right) \\
\partial_{t} N_{2}=-\frac{2}{\eta} \operatorname{Im}\left(E_{L} Q_{1}^{*}\right)-4 c_{4} \operatorname{Im}\left(E_{S} Q_{3}^{*}\right)
\end{array}\right.
$$

By usual techniques, one can shows that, for an initial Cauchy data in $H^{s}\left(\mathbb{R}^{2}\right)$ with $s>1$, there exists a unique solution of $(\mathbf{M})$ in $\mathcal{C}^{0}\left(\left[0, T_{0}\right], H^{s}\left(\mathbb{R}^{2}\right)\right) \cap$ $\mathcal{C}^{1}\left(\left[0, T_{0}\right], H^{s-2}\left(\mathbb{R}^{2}\right)\right)$. We show below $T_{0}=+\infty$ (Theorem 2$)$.

Now, in view of the relations $\mathcal{R}$, we take an initial data that corresponds to the above approximate solution. We call it, as usual, "well-prepared" (since it corresponds to the initial data of the WKB solution):

$$
\left[\begin{array}{c}
\left(B_{1} ; B_{2} ; E ; Q_{1} ; Q_{2} ; Q_{3} ; N_{1} ; N_{2}\right)(t=0)=\left(0 ;-E_{L}^{0} e^{i x / \epsilon}-E_{S}^{0} e^{i b x / \epsilon}+c . c ;\right. \\
\left.E_{L}^{0} e^{i x / \epsilon}+E_{S}^{0} e^{i b x / \epsilon}+c . c ; Q_{1}^{0} e^{i x / \epsilon} ; Q_{2}^{0} e^{i x(1-b) / \epsilon} ; Q_{3}^{0} e^{-i x b / \epsilon} ; N_{1}^{0} ; N_{2}^{0}\right) .
\end{array}\right.
$$

To define the approximate solution, let $\left(E_{L} ; E_{S} ; Q_{1,2,3} ; N_{1,2}\right)$ be the solution of the initial value problem for $(\mathbf{M})$ with respect to the initial data $\left(E_{L}^{0} ; E_{S}^{0} ; Q_{1,2,3}^{0} ; N_{1,2}^{0}\right)$.

For the fundamental laser oscillation $e^{i \theta_{1}}$, we define an approximate solution as follows:

$$
\left\{\begin{aligned}
u_{1,0}^{\epsilon} & =\left(0,-E_{L}, E_{L}, Q_{1}, 0,0,0,0\right)+\sqrt{\epsilon}\left(-i \sqrt{L} \partial_{y} E_{L}, 0,0,0,0,0,0,0\right) \\
& +\epsilon\left(0, i\left(\partial_{t}+\partial_{x}\right) E_{L}, 0,0,0, \frac{1}{1+b}\left[\frac{E_{S} Q_{2}}{\eta}-c_{4} E_{L} N_{2}\right], 0,0\right) \\
u_{-1,0}^{\epsilon} & =\left(0,-E_{L}^{*}, E_{L}^{*}, 0,0,0,0,0\right)+\sqrt{\epsilon}\left(i \sqrt{L} \partial_{y} E_{L}^{*}, 0,0,0,0,0,0,0\right) \\
& +\epsilon\left(0,-i\left(\partial_{t}+\partial_{x}\right) E_{L}^{*}, 0,-\frac{1}{2 \eta} E_{L}^{*}-\frac{c_{2}}{2} E_{L}^{*} N_{1}, 0, \frac{c_{4}}{1-b} E_{L}^{*} N_{2}, 0,0\right)
\end{aligned}\right.
$$


For the Raman oscillation $e^{i \theta_{2}}$, we define an approximate solution as follows:

$$
\left\{\begin{aligned}
u_{0,1}^{\epsilon} & =\left(0,-E_{S}, E_{S}, 0,0,0,0,0\right)+\sqrt{\epsilon}\left(-i \frac{\sqrt{L}}{b} \partial_{y} E_{S}, 0,0,0,0,0,0,0\right) \\
& +\epsilon\left(0, \frac{i}{b}\left(\partial_{t}+\partial_{x}\right) E_{S}, 0, \frac{1}{1-b}\left[\frac{E_{S}}{\eta}-c_{2} E_{S} N_{1}\right], 0,-\frac{c_{4}}{2 b} E_{S} N_{2}, 0,0\right), \\
u_{0,-1}^{\epsilon} & =\left(0,-E_{S}^{*}, E_{S}^{*}, 0,0, Q_{3}^{*}, 0,0\right)+\sqrt{\epsilon}\left(i \frac{\sqrt{L}}{b} \partial_{y} E_{S}^{*}, 0,0,0,0,0,0,0\right) \\
& +\epsilon\left(0,-\frac{i}{b}\left(\partial_{t}+\partial_{x}\right) E_{S}^{*}, 0, \frac{1}{1+b}\left[\frac{E_{S}^{*}}{\eta}-c_{2} E_{S}^{*} N_{1}+c_{4} E_{L}^{*} Q_{2}\right], 0,0,0,0\right) .
\end{aligned}\right.
$$

In order to define the amplitude associated with the quantic variables $\left(Q_{i}, N_{i}\right)$, we set

$$
\left\{\begin{aligned}
u_{0,0}^{\epsilon} & =\left(0,0,0,0,0,0, N_{1}, N_{2}\right)+\epsilon\left(0,0,0,0, \frac{1}{1-b}\left[-c_{2} E_{S} Q_{3}^{*}+c_{4} E_{L}^{*} Q_{1}\right], 0,0,0\right), \\
u_{1,-1}^{\epsilon} & =\left(0,0,0,0, Q_{2}, 0,0,0\right) \\
& +\epsilon\left(0,0,0,0,0,0, \frac{2}{1-b}\left[\frac{4}{\eta} E_{S}^{*} Q_{1}-2 c_{4} E_{L} Q_{3}^{*}\right], \frac{2}{1-b}\left[\frac{2}{\eta} E_{S}^{*} Q_{1}-4 c_{4} E_{L} Q_{3}^{*}\right]\right) .
\end{aligned}\right.
$$

The key point of this construction is that the leading terms are given by the solution of the system $(\mathbf{M})$. The $\mathcal{O}(\sqrt{\epsilon})$ and $\mathcal{O}(\epsilon)$ terms are correctors deduced from equations $(2.17)-(2.32)$.

Finally, we must define higher order harmonics which are nonlinear corrections of order $\epsilon$.

$$
\left\{\begin{aligned}
u_{2,-1}^{\epsilon}= & \epsilon\left(0,0,0, \frac{c_{4}}{(b-1)} Q_{2} E_{L}, 0, \frac{-1}{(b-1) \eta} Q_{2} E_{L}, 0,0\right) \\
u_{1,-2}^{\epsilon}= & \epsilon\left(0,0,0, \frac{c_{4}}{2 b} Q_{2} E_{S}^{*}, 0, \frac{-1}{2 b \eta} Q_{2} E_{S}^{*}, 0,0\right) \\
u_{1,1}^{\epsilon}= & \epsilon\left(0,0,0,0, \frac{-c_{4}}{2 b} Q_{1} E_{S}, 0, \frac{2}{1+b}\left[\frac{4}{\eta} E_{S} Q_{1}+2 c_{4} E_{L} Q_{3}\right]\right. \\
& \left.\frac{1}{1+b}\left[\frac{4}{\eta} E_{S} Q_{1}+2 c_{4} E_{L} Q_{3}\right]\right) \\
u_{-1,1}^{\epsilon}= & \epsilon\left(0,0,0,0, \frac{c_{4}}{2(1-b)} E_{S}^{*} Q_{1}, 0, \frac{-4}{1-b}\left[\frac{2}{\eta} E_{S}^{*} Q_{1}-\frac{2}{\eta} E_{S} Q_{1}^{*}+c_{4} E_{L}^{*} Q_{3}\right]\right. \\
& \left.\frac{-4}{1-b}\left[\frac{1}{\eta} E_{S}^{*} Q_{1}-\frac{1}{\eta} E_{S} Q_{1}^{*}+2 c_{4} E_{L}^{*} Q_{3}\right]\right) \\
u_{-1,-1}^{\epsilon}= & \epsilon\left(0,0,0,0, \frac{-c_{2}}{2} E_{L}^{*} Q_{3}^{*}, 0, \frac{-2}{1+b}\left[\frac{4}{\eta} E_{S}^{*} Q_{1}^{*}-2 c_{4} E_{L}^{*} Q_{3}^{*}\right]\right. \\
& \left.\frac{-2}{1+b}\left[\frac{2}{\eta} E_{S}^{*} Q_{1}^{*}-4 c_{4} E_{L}^{*} Q_{3}^{*}\right]\right) \\
u_{2,0}^{\epsilon}= & \epsilon\left(0,0,0,0, \frac{-c_{4}}{(1+b)} Q_{1} E_{L}, 0, \frac{4}{\eta} E_{L} Q_{1}, \frac{2}{\eta} E_{L} Q_{1}\right) \\
u_{0,-2}^{\epsilon}= & \epsilon\left(0,0,0,0, \frac{-c_{2}}{(1+b)} E_{S}^{*} Q_{3}^{*}, 0, \frac{2 c_{4}}{b} E_{S}^{*} Q_{3}^{*}, \frac{4 c_{4}}{b} E_{S}^{*} Q_{3}^{*}\right) \\
u_{-2,0}^{\epsilon}= & \epsilon\left(0,0,0,0,0,0, \frac{4}{\eta} E_{L}^{*} Q_{1}^{*}, \frac{2}{\eta} E_{L}^{*} Q_{1}^{*}\right) \\
u_{0,2}^{\epsilon}= & \epsilon\left(0,0,0,0,0,0, \frac{2 c_{4}}{b} E_{S} Q_{3}, \frac{4 c_{4}}{b} E_{S} Q_{3}\right)
\end{aligned}\right.
$$

The approximate solution is now defined by

$$
\mathbf{u}_{\text {app }}^{\epsilon}\left(t, x, y, \theta_{1}, \theta_{2}\right)=\sum_{p_{i} \in \mathcal{D}} u_{p_{1}, p_{2}}^{\epsilon}(t, x, y) e^{i\left(p_{1} \theta_{1}+p_{2} \theta_{2}\right)}=u_{1,0}^{\epsilon} e^{i \theta_{1}}+\cdots+u_{0,2}^{\epsilon} e^{2 i \theta_{2}},
$$

where $\mathcal{D}$ denotes the set of all the harmonics defined above. 


\subsection{Error estimate}

To prove the stability of the asymptotic expansion, we evaluate the remainder defined by

$$
\mathbf{r}^{\epsilon}\left(t, x, y, \theta_{1}, \theta_{2}\right)=\mathbf{r}_{\mathbf{1}}{ }^{\epsilon}+\sqrt{\epsilon} \mathbf{r}_{\mathbf{2}}{ }^{\epsilon}+\epsilon \mathbf{r}_{\mathbf{3}}{ }^{\epsilon}=\widetilde{\mathcal{L}}(\partial)\left(\mathbf{u}_{\mathbf{a p p}}^{\epsilon}\right)-f\left(\mathbf{u}_{\mathbf{a p p}}^{\epsilon}\right),
$$

where $\widetilde{\mathcal{L}}(\partial)$ is the linear symmetric hyperbolic operator $\mathcal{L}(\partial)$ written in the profile variables:

$$
\widetilde{\mathcal{L}}(\partial):=\left(-\frac{1}{\epsilon} \partial_{\theta_{1}}-\frac{b}{\epsilon} \partial_{\theta_{2}}+\partial_{t}\right)+A_{1}\left(\frac{1}{\epsilon} \partial_{\theta_{1}}+\frac{b}{\epsilon} \partial_{\theta_{2}}+\partial_{x}\right)+A_{2} \partial_{y}+\frac{L_{0}}{\epsilon},
$$

where the matrix $A_{1,2}$ and $L_{0}$ are defined by (1).

Proposition 2.1. For all $t<+\infty$, we have the following estimate:

$$
\left\|\mathbf{r}^{\epsilon}\right\|_{L^{\infty}\left([0, t], H^{s-4}\left(\mathbb{R}^{2} \times \mathbb{T}^{2}\right)\right)} \leq C_{1}(t) \sqrt{\epsilon} \quad\left(\text { where } C_{1}(t) \text { is } L_{l o c}^{\infty}\left(\mathbb{R}_{t}^{+}\right)\right) .
$$

Proof. Direct computations show that the remainder component $\mathbf{r}_{1}{ }^{\epsilon}=0$.

The $\mathbf{r}_{\mathbf{2}}{ }^{\epsilon}$ component come from Maxwell's part of the system $(\mathbf{S})$ and involve the terms $\partial_{t y}\left(E_{L}+E_{S}\right)$ and $\left(\partial_{t t}+\partial_{t x}\right)\left(E_{L}+E_{S}\right)$.

For the last part $\mathbf{r}_{\mathbf{3}}{ }^{\epsilon}$ of the remainder, the main terms are $\left(\partial_{t t}+\partial_{t x}\right)\left(E_{L}+E_{S}\right)$. Finally, the regularity of $E_{S}$ and $E_{L}$ gives the result.

Now, we must prove that the Cauchy problem for $(\mathbf{S})$ has a unique solution defined on an interval $[0, T(\eta)]$ with $T(\eta)$ independent of $\epsilon$. For this, it suffices to see that the hyperbolic operator $\mathcal{L}(\partial)$ defined by $(1)$ generates a unitary group (which absorbs all the terms in $1 / \epsilon$ ). A fixed-point theorem achieves the proof. In fact, as for $(\mathbf{M})$, one can see that we have $T(\eta)=+\infty$ (see Theorem 2).

Let us denote by $(\widetilde{\mathbf{S}})$ the system $(\mathbf{S})$ in the profile variables:

$$
(\widetilde{\mathbf{S}}): \widetilde{\mathcal{L}}(\partial) \mathbf{u}=f(\epsilon, \eta, \mathbf{u}),
$$

where $\widetilde{\mathcal{L}}(\partial)$ and $f(\epsilon, \eta, \mathbf{u})$ are defined by $(2.35)$ and $(1)$.

The same argument as above implies the existence of a unique solution defined on an interval $[0, \widetilde{T}(\eta)]$ with $\widetilde{T}(\eta)$ independent of $\epsilon$ (in fact, we have $\widetilde{T}(\eta)=+\infty$ ). Finally, let us define the following Cauchy data

$$
\left[\begin{array}{c}
\left(\mathbf{B}_{\mathbf{1}} ; \mathbf{B}_{\mathbf{2}} ; \mathbf{E} ; \mathbf{Q}_{\mathbf{1}} ; \mathbf{Q}_{\mathbf{2}} ; \mathbf{Q}_{\mathbf{3}} ; \mathbf{N}_{\mathbf{1}} ; \mathbf{N}_{\mathbf{2}}\right)(t=0)=\left(0 ;-E_{L}^{0} e^{i \theta_{1}}-E_{S}^{0} e^{i \theta_{2}}+c . c ;\right. \\
\left.E_{L}^{0} e^{i \theta_{1}}+E_{S}^{0} e^{i \theta_{2}}+\text { c.c } ; Q_{1}^{0} e^{i \theta_{1}} ; Q_{2}^{0} e^{i\left(\theta_{1}-\theta_{2}\right)} ; Q_{3}^{0} e^{-i \theta_{2}} ; N_{1}^{0} ; N_{2}^{0}\right)
\end{array}\right.
$$

which is the Cauchy data (2.33) in the profile variables.

We can now state the expected stability result 
Theorem 2.1. Let $s>6$.

Let $\mathbf{u}^{\epsilon}$ be the solution of the system $(\widetilde{\mathbf{S}})$ in $\mathcal{C}^{0}\left(\left[0 ;+\infty\left[, H^{s}\left(\mathbb{R}^{2} \times \mathbb{T}^{2}\right)\right)\right.\right.$ associated with the Cauchy data (2.36).

Let us denote by $\mathbf{u}_{\text {app }}^{\mathbf{1}}$ the $\mathcal{O}(1)$-term of $\mathbf{u}_{\text {app }}^{\epsilon}$ in (2.34):

$\left(0 ;-E_{L} e^{i \theta_{1}}-E_{S} e^{i \theta_{2}}+c . c ; E_{L} e^{i \theta_{1}}+E_{S} e^{i \theta_{2}}+c . c ; Q_{1} e^{i \theta_{1}} ; Q_{2} e^{i\left(\theta_{1}-\theta_{2}\right)} ; Q_{3}^{*} e^{-i \theta_{2}} ; N_{1} ; N_{2}\right)$.

Then, for any fixed $T>0$, there exists a constant $C(T)>0$ independant of $\epsilon$ such that for $\epsilon$ small enough:

$$
\left\|\mathbf{u}^{\epsilon}-\mathbf{u}_{\text {app }}^{\mathbf{1}}\right\|_{L^{\infty}\left([0, T] \times \mathbb{T}^{2} \times \mathbb{R}^{2}\right)} \leq C(T) \sqrt{\epsilon} .
$$

Corollary 2.1. Let $s>6$.

Let $u^{\epsilon}$ be the solution of the system $(\mathbf{S})$ in $\mathcal{C}^{0}\left(\left[0,+\infty\left[, H^{s}\left(\mathbb{R}^{2}\right)\right)\right.\right.$ associated with the Cauchy data (2.33).

Let us denote by $u_{a p p}^{1}$ the trace on $\left(\theta_{1}=\frac{x-t}{\epsilon} ; \theta_{2}=b \frac{x-t}{\epsilon}\right)$ of $\mathbf{u}_{a p p}^{\mathbf{1}}$.

Then, with the notation of Theorem 1, we have the following estimate:

$$
\left\|u^{\epsilon}-u_{a p p}^{1}\right\|_{L^{\infty}\left([0, T] \times \mathbb{R}^{2}\right)} \leq C(T) \sqrt{\epsilon} .
$$

Proof. We use the classical energy estimate for the linear symmetric hyperbolic operator $\widetilde{\mathcal{L}}$ defined above. Let $\mathbf{w}^{\epsilon}=\mathbf{u}^{\epsilon}-\mathbf{u}_{\text {app }}^{\epsilon}$ and let us consider an arbitrary $T>0$.

For $\epsilon$ sufficiently small, we have $\left\|\mathbf{w}^{\epsilon}(0)\right\|_{H^{s-4}} \leq C \sqrt{\epsilon} \leq 1$. So, by continuity, we can define:

$$
T^{*}(\epsilon)=\sup \left\{t<T \text {, such that }\left\|\mathbf{w}^{\epsilon}\right\|_{L^{\infty}\left([0, t] ; H^{s-4}\right)} \leq 2\right\} .
$$

For $0 \leq t \leq T^{*}(\epsilon)$, the energy estimate for $\widetilde{\mathcal{L}}$ reads:

$$
\begin{aligned}
\left\|\mathbf{w}^{\epsilon}(t)\right\|_{L^{\infty}\left([0, t] ; H^{s-4}\right)} \leq & \left\|\mathbf{w}^{\epsilon}(0)\right\|_{H^{s-4}}+2 \int_{0}^{t}\left\|\widetilde{\mathcal{L}} \mathbf{w}^{\epsilon}\left(t^{\prime}\right)\right\|_{H^{s-4}} d t^{\prime} \\
\leq & \left\|\mathbf{w}^{\epsilon}(0)\right\|_{H^{s-4}}+2 \int_{0}^{t}\left\|\mathbf{r}^{\epsilon}\left(t^{\prime}\right)\right\|_{H^{s-4}} d t^{\prime} \\
& +C_{2} \int_{0}^{t}\left\|\mathbf{w}^{\epsilon}\left(t^{\prime}\right)\right\|_{H^{s-4}} d t^{\prime}
\end{aligned}
$$

(the last inequality is due to the polynomial character of the nonlinearity). Using the estimate in $\mathcal{O}(\sqrt{\epsilon})$ of the remainder $\mathbf{r}^{\epsilon}$, we obtain:

$$
\left\|\mathbf{w}^{\epsilon}(t)\right\|_{L^{\infty}\left([0, t] ; H^{s-4}\right)} \leq C \sqrt{\epsilon}+T C_{1} \sqrt{\epsilon}+2 C_{2} \int_{0}^{t}\left\|\mathbf{w}^{\epsilon}\left(t^{\prime}\right)\right\|_{H^{s-4}} d t^{\prime} .
$$

where $C_{1}$ denotes the $L^{\infty}([0 ; T])$-norm of the function $C_{1}(t)$ that appears in the Proposition 1. For $\epsilon$ sufficiently small, Gronwall's lemma yields $\left\|\mathbf{w}^{\epsilon}(t)\right\|_{L^{\infty}\left([0, t] ; H^{s-4}\right)} \leq$ 1 , for all $t \leq T$.

Therefore, we have $T^{*}(\epsilon)=T$ and the result follows from the injection $H^{s-4}\left(\mathbb{R}^{2} \times\right.$ $\left.\mathbb{T}^{2}\right) \hookrightarrow L^{\infty}\left(\mathbb{R}^{2} \times \mathbb{T}^{2}\right)$ which is valid when $s>6$ 


\section{Comment:}

The key point of our asymptotic analysis is related to the evolution of the electric field:

$$
E(t, x, y) \sim E_{L} e^{i \frac{x-t}{\epsilon}}+E_{S} e^{i b \frac{x-t}{\epsilon}}+c . c+\mathcal{O}(\sqrt{\epsilon}) .
$$

This means that the oscillatory components $\left(E_{L} ; E_{S}\right)$ of the initial pulse propagate.

Nevertheless, this asymptotic behavior involves an error which is only in $\mathcal{O}(\sqrt{\epsilon})$. This is numerically interesting for the physical situations that we have considered since $\epsilon$ is really small $\left(\sim 10^{-8}\right)$ but it cannot be of any interest for ultrashort (picoseconds or less) pulses.

Finally, let us emphasize the fact that our analysis extends whitout problems for any multi-level Maxwell-Bloch system. In particular, this allows the justification of the formal asymptotic performed in Colin-Nkonga[6] for the two-level system:

$$
\left(\mathbf{S}_{\mathbf{2}}\right)\left\{\begin{array}{l}
\partial_{t} B_{1}+\frac{1}{\epsilon_{1}} \partial_{y} E=0, \\
\partial_{t} B_{2}-\partial_{x} E=0, \\
\partial_{t} E-\left(\partial_{x} B_{2}-\frac{1}{\epsilon_{1}} \partial_{y} B_{1}\right)=i c_{1}\left(1+\frac{\epsilon}{\eta}\right) Q_{1}+\text { c.c, } \\
\partial_{t} Q_{1}=-i\left(\frac{1}{\epsilon}+\frac{1}{\eta}\right) Q_{1}+\frac{i}{\eta} E-i c_{2} E N_{1}, \\
\partial_{t} N_{1}=\frac{4}{\eta} \operatorname{Im}\left(Q_{1}\right) E .
\end{array}\right.
$$

Indeed, introducing the system:

$$
\left(\mathbf{M}_{2}\right)\left\{\begin{array}{l}
\left(\partial_{t}+\partial_{x}\right) E_{L}-i \frac{L}{2} \partial_{y^{2}}^{2} E_{L}=i \frac{c_{1}}{2} \eta Q_{1}, \\
\partial_{t} Q_{1}=-\frac{i}{\eta} Q_{1}+\frac{i}{\eta} E_{L}-i c_{2} E_{L} N_{1}, \\
\partial_{t} N_{1}=-\frac{4}{\eta} \operatorname{Im}\left(E_{L} Q_{1}^{*}\right)
\end{array}\right.
$$

the following asymptotic result holds:

Theorem 2.2. Let $s>5$.

Let us denote by $u^{\epsilon}=\left(B_{1}^{\epsilon} ; B_{2}^{\epsilon} ; E^{\epsilon} ; Q_{1}^{\epsilon} ; N_{1}^{\epsilon}\right)$ the solution of the system $\left(\mathbf{S}_{\mathbf{2}}\right)$ in $\mathcal{C}^{0}\left(\left[0,+\infty\left[, H^{s}\left(\mathbb{R}^{2}\right)\right)\right.\right.$ associated with the Cauchy data:

$$
u^{\epsilon}(t=0)=\left(0 ;-E_{L}^{0} e^{i \frac{x}{\epsilon}}+c . c ; E_{L}^{0} e^{i \frac{x}{\epsilon}}+c . c ; Q_{1}^{0} e^{i \frac{x}{\epsilon}} ; N_{1}^{0}\right) .
$$

Let $\left(E_{L} ; Q_{1} ; N_{1}\right)$ be the solution of $\left(\mathbf{M}_{2}\right)$ in $\mathcal{C}^{0}\left(\left[0, \infty\left[, H^{s}\left(\mathbb{R}^{2}\right)\right)\right.\right.$ associated with the Chauchy data $\left(E_{L}^{0}, Q_{1}^{0}, N_{1}^{0}\right)$.

Let $u_{a p p}^{1}$ be defined by:

$$
u_{\text {app }}^{1}=\left(0 ;-E_{L} e^{i \frac{x-t}{\epsilon}}+c . c ; E_{L} e^{i \frac{x-t}{\epsilon}}+c . c ; Q_{1} e^{i \frac{x-t}{\epsilon}} ; N_{1}\right) .
$$


Then,for any fixed $T>0$, there exists a constant $C(T)>0$ independant of $\epsilon$ such that for $\epsilon$ small enough:

$$
\left\|u^{\epsilon}-u_{a p p}^{1}\right\|_{L^{\infty}\left([0, T] \times \mathbb{R}^{2}\right)} \leq C(T) \sqrt{\epsilon} .
$$

\section{On the global solvability of systems (S) and (M)}

We will use the very particular form of systems $(\mathbf{S})$ and $(\mathbf{M})$ in order to obtain some global existence results. In one or two dimension, our proofs are very simple. For the 3D Maxwell-Bloch system, we apply E.Dumas's result [9] and for the Schrödinger-Bloch system, we have an original result.

\subsection{The cases $\mathbb{R}$ and $\mathbb{R}^{2}$}

Let us introduce the following general form

$$
\left(\mathbf{R}^{\mathbf{n}}\right)\left\{\begin{array}{l}
\partial_{t} \mathbf{U}_{\mathbf{1}}+i P(D) \mathbf{U}_{\mathbf{1}}=i F\left(\mathbf{U}_{\mathbf{2}}\right) ; \\
\partial_{t} \mathbf{U}_{\mathbf{2}}=i H \mathbf{U}_{\mathbf{2}}+A\left(\mathbf{U}_{\mathbf{1}}\right) \mathbf{U}_{\mathbf{2}} ;
\end{array}\right.
$$

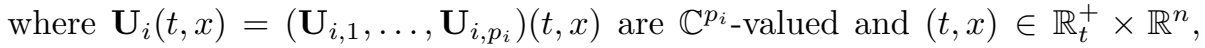
$H$ is a self-adjoint matrix, $P(D)$ is an arbitrary differential operator with real symbol of order $m, F$ is a polynomial map which vanishes at the origin, the map $u \longrightarrow A(u)$ is linear and $A\left(\mathbf{U}_{1}\right)$ is skew-adjoint in $\mathcal{M}^{p_{2}}(\mathbb{C})$.

We can state the following result:

Theorem 3.1. Let us consider the system $\left(\mathbf{R}^{\mathbf{n}}\right)$ with $n \leq 2$. For any initial datum $\mathbf{U}^{\mathbf{0}}$ in $H^{s}\left(\mathbb{R}^{n}\right)$ with $s>n / 2$, the system $\left(\mathbf{R}^{\mathbf{n}}\right)$ has a unique solution in $\mathcal{C}^{0}\left(\mathbb{R}_{t}^{+}, H^{s}\left(\mathbb{R}^{n}\right)\right) \cap \mathcal{C}^{1}\left(\mathbb{R}_{t}^{+}, H^{s-m}\left(\mathbb{R}^{n}\right)\right)$.

Moreover, one has $\mathbf{U}_{\mathbf{2}}$ in $L^{\infty}\left(\mathbb{R}_{t}^{+} ; L_{x}^{2}\left(\mathbb{R}^{n}\right)\right) \cap L^{\infty}\left(\mathbb{R}_{t}^{+} \times \mathbb{R}_{x}^{n}\right)$.

Proof. Usual techniques prove the existence of a unique solution for $t \in\left[0 ; T_{\max }[\right.$. We prove that $T_{\max }=+\infty$.

The key point is that since $i H+A\left(\mathbf{U}_{\mathbf{1}}\right)$ is skew-adjoint, we have:

$$
\operatorname{Re}\left\langle\left[i H+A\left(\mathbf{U}_{\mathbf{1}}\right)\right] \mathbf{U} ; \mathbf{U}\right\rangle_{\mathbb{C}^{p_{2}}}=0 .
$$

So, for every $(t, x) \in \mathbb{R}^{+} \times \mathbb{R}^{n}$ :

$$
R e\left\langle\partial_{t} \mathbf{U}_{\mathbf{2}} ; \mathbf{U}_{\mathbf{2}}\right\rangle_{\mathbb{C}^{p_{2}}}=0 .
$$

Therefore

$$
\partial_{t}\left|\mathbf{U}_{\mathbf{2}}\right|_{\mathbb{C}^{p_{2}}}^{2}=0 \text { and so }\left\|\mathbf{U}_{\mathbf{2}}(t)\right\|_{L^{\infty}}=\left\|\mathbf{U}_{\mathbf{2}}^{\mathbf{0}}\right\|_{L^{\infty}} .
$$

This implies $\mathbf{U}_{\mathbf{2}} \in L^{\infty}\left(\mathbb{R}_{t}^{+} \times \mathbb{R}^{n}\right) \cap L^{\infty}\left(\mathbb{R}_{t}^{+} ; L^{2}\left(\mathbb{R}^{n}\right)\right)$. Now, since $F$ is smooth and $F(0)=0$, these $L^{\infty}\left(\mathbb{R}^{n+1}\right)$ bounds imply that

$$
\left\|F\left(\mathbf{U}_{\mathbf{2}}\right)\right\|_{H^{s}} \leq C_{0}\left\|\mathbf{U}_{\mathbf{2}}\right\|_{H^{s}},
$$


where the constant $C_{0}$ depends on $\left\|\mathbf{U}_{\mathbf{2}}^{0}\right\|_{L^{\infty}\left(\mathbb{R}^{n}\right)}$.

Thus, since $H^{s} \cap L^{\infty}$ is an algebra, we have:

$$
\partial_{t}\left\|\mathbf{U}_{\mathbf{1}}\right\|_{H^{s}}^{2} \leq C_{0}\left\|\mathbf{U}_{\mathbf{2}}\right\|_{H^{s}}\left\|\mathbf{U}_{\mathbf{1}}\right\|_{H^{s}} \leq C_{0}\left(\left\|\mathbf{U}_{\mathbf{2}}\right\|_{H^{s}}^{2}+\left\|\mathbf{U}_{\mathbf{1}}\right\|_{H^{s}}^{2}\right) .
$$

It remains to control $\left\|\mathbf{U}_{\mathbf{2}}\right\|_{H^{1}}$. (33) applied to

$$
\begin{aligned}
\left\langle\partial_{t} \partial_{x} \mathbf{U}_{\mathbf{2}} ; \partial_{x} \mathbf{U}_{\mathbf{2}}\right\rangle_{\mathbb{C}^{p_{2}}} & =\left\langle i H \partial_{x} \mathbf{U}_{\mathbf{2}} ; \partial_{x} \mathbf{U}_{\mathbf{2}}\right\rangle_{\mathbb{C}^{p_{2}}}+\left\langle A\left(\partial_{x} \mathbf{U}_{\mathbf{1}}\right) \mathbf{U}_{\mathbf{2}} ; \partial_{x} \mathbf{U}_{\mathbf{2}}\right\rangle_{\mathbb{C}^{p_{2}}} \\
& +\left\langle A\left(\mathbf{U}_{\mathbf{1}}\right) \partial_{x} \mathbf{U}_{\mathbf{2}} ; \partial_{x} \mathbf{U}_{\mathbf{2}}\right\rangle_{\mathbb{C}^{p_{2}}},
\end{aligned}
$$

leads to

$$
\partial_{t}\left\|\partial_{x} \mathbf{U}_{\mathbf{2}}\right\|_{L^{2}}^{2} \leq\left\|A\left(\partial_{x} \mathbf{U}_{\mathbf{1}}\right)\right\|_{L^{2}}\left\|\mathbf{U}_{\mathbf{2}}\right\|_{L^{\infty}}\left\|\partial_{x} \mathbf{U}_{\mathbf{2}}\right\|_{L^{2}} \leq C_{0}\left\|\partial_{x} \mathbf{U}_{\mathbf{1}}\right\|_{L^{2}}\left\|\partial_{x} \mathbf{U}_{\mathbf{2}}\right\|_{L^{2}},
$$

as $u \longrightarrow A(u)$ is linear. Hence

$$
\partial_{t}\left\|\partial_{x} \mathbf{U}_{\mathbf{2}}\right\|_{L^{2}}^{2} \leq C_{0}\left(\left\|\partial_{x} \mathbf{U}_{\mathbf{1}}\right\|_{L^{2}}^{2}+\left\|\partial_{x} \mathbf{U}_{\mathbf{2}}\right\|_{L^{2}}^{2}\right) .
$$

(34), (35) and Gronwall's lemma allow to control the $H^{1}$ norm of the solution and gives the conclusion for the $1 \mathrm{D}$ case. This is not sufficient for the $2 \mathrm{D}$ case since this $H^{1}$ estimate does not give the $L^{\infty}$ control of $\mathbf{U}_{\mathbf{1}}$. Nevertheless, using $\operatorname{Re}\left\langle A\left(\mathbf{U}_{\mathbf{1}}\right) \partial_{x}^{2} \mathbf{U}_{\mathbf{2}} ; \partial_{x}^{2} \mathbf{U}_{\mathbf{2}}\right\rangle_{\mathbb{C}^{p_{2}}}=0$, we have:

$$
\begin{aligned}
\operatorname{Re}\left\langle\partial_{t} \partial_{x}^{2} \mathbf{U}_{\mathbf{2}} ; \partial_{x}^{2} \mathbf{U}_{\mathbf{2}}\right\rangle_{\mathbb{C}^{p_{2}}} & =\operatorname{Re}\left\langle A\left(\partial_{x}^{2} \mathbf{U}_{\mathbf{1}}\right) \mathbf{U}_{\mathbf{2}} ; \partial_{x}^{2} \mathbf{U}_{\mathbf{2}}\right\rangle_{\mathbb{C}^{p_{2}}} \\
& +2 \operatorname{Re}\left\langle A\left(\partial_{x} \mathbf{U}_{\mathbf{1}}\right) \partial_{x} \mathbf{U}_{\mathbf{2}} ; \partial_{x}^{2} \mathbf{U}_{\mathbf{2}}\right\rangle_{\mathbb{C}^{p_{2}}} .
\end{aligned}
$$

The first term is good since it involves $\left\|\mathbf{U}_{\mathbf{2}}\right\|_{\infty}$. In order to control the second term, we use Hölder and a Gagliardo-Nirenberg inequality:

$$
\begin{aligned}
\int_{R^{n}} \operatorname{Re}\left\langle A\left(\partial_{x} \mathbf{U}_{\mathbf{1}}\right) \partial_{x} \mathbf{U}_{\mathbf{2}} ; \partial_{x}^{2} \mathbf{U}_{\mathbf{2}}\right\rangle & \leq\left\|\partial_{x} \mathbf{U}_{\mathbf{1}}\right\|_{L^{4}}\left\|\partial_{x} \mathbf{U}_{\mathbf{2}}\right\|_{L^{4}}\left\|\partial_{x}^{2} \mathbf{U}_{\mathbf{2}}\right\|_{L^{2}}, \\
& \leq\left\|\mathbf{U}_{\mathbf{1}}\right\|_{L^{\infty}}^{1 / 2}\left\|\partial_{x}^{2} \mathbf{U}_{\mathbf{1}}\right\|_{L^{2}}^{1 / 2}\left\|\mathbf{U}_{\mathbf{2}}\right\|_{L^{\infty}}^{1 / 2}\left\|\partial_{x}^{2} \mathbf{U}_{\mathbf{2}}\right\|_{L^{2}}^{3 / 2}, \\
& \leq C_{0}\left\|\mathbf{U}_{\mathbf{1}}\right\|_{L^{\infty}}^{1 / 2}\left\|\partial_{x}^{2} \mathbf{U}_{\mathbf{1}}\right\|_{L^{2}}^{1 / 2}\left\|\partial_{x}^{2} \mathbf{U}_{\mathbf{2}}\right\|_{L^{2}}^{3 / 2} .
\end{aligned}
$$

We just need to control $\left\|\mathbf{U}_{\mathbf{1}}\right\|_{\infty}$. In this view, recall that we already have a control on $\left\|\mathbf{U}_{\mathbf{1}}\right\|_{H^{1}}$, so we can use the following 2D Sobolev inequality (due to Brezis and Gallouët[5]):

$$
\left\|\mathbf{U}_{\mathbf{1}}\right\|_{\infty} \leq\left\|\mathbf{U}_{\mathbf{1}}\right\|_{H^{1}}\left(\ln \left(1+\left\|\mathbf{U}_{\mathbf{1}}\right\|_{H^{2}}^{2}\right)\right)^{1 / 2}+\text { Cte. }
$$

Finally, using (34) for $s=2$, we obtain:

$$
\partial_{t}\|\mathbf{U}\|_{H^{2}}^{2} \leq C_{0}\left(1+\ln \left(1+\|\mathbf{U}\|_{H^{2}}^{2}\right)\right)\|\mathbf{U}\|_{H^{2}}^{2},
$$

where $\|\mathbf{U}\|_{H^{2}}=\left\|\mathbf{U}_{\mathbf{1}}\right\|_{H^{2}}+\left\|\mathbf{U}_{\mathbf{2}}\right\|_{H^{2}}$.

This estimate leads to $\|\mathbf{U}\|_{H^{2}} \leq \alpha e^{e^{\beta t}}$ and the conclusion follows. 


\section{Remark 1:}

Both systems (S) and (M) have the generic form $\left(\mathbf{R}^{2}\right)$. Indeed, instead of using the Bloch variables, we can write these systems in the variables $\left(a_{0}, a_{1}, a_{1^{\prime}}\right)$ to obtain:

$$
\left(\mathbf{S}^{\prime}\right)\left\{\begin{array}{l}
\partial_{t} B_{1}+\partial_{y} E=0 \\
\partial_{t} B_{2}-\partial_{x} E=0 \\
\partial_{t} E-\left(\partial_{x} B_{2}-\partial_{y} B_{1}\right)=c_{1} a_{1} a_{0}^{*}+c_{2} a_{1}^{*} a_{1^{\prime}}+c . c \\
\partial_{t} a_{0}=i c_{3} E a_{1} \\
\partial_{t} a_{1}=-i \omega_{1} a_{1}+i c_{3} E a_{0}+i c_{4} E a_{1^{\prime}}, \\
\partial_{t} a_{1^{\prime}}=-i \omega_{1^{\prime}} a_{1^{\prime}}+i c_{4} E a_{1},
\end{array}\right.
$$

and

$$
\left(\mathbf{M}^{\prime}\right)\left\{\begin{array}{l}
\left(\partial_{t}+\partial_{x}\right) E_{S}-i \frac{L}{2 b} \partial_{y^{2}}^{2} E_{S}=i c_{1} a_{1} a_{1^{\prime}}^{*} \\
\left(\partial_{t}+\partial_{x}\right) E_{L}-i \frac{L}{2} \partial_{y^{2}}^{2} E_{L}=i c_{2} a_{1} a_{0}^{*} \\
\partial_{t} a_{0}=i c_{3} E_{L}^{*} a_{1} \\
\partial_{t} a_{1}=-\frac{i}{\eta} a_{1}+i c_{3} E_{L} a_{0}+i c_{4} E_{S} a_{1^{\prime}} \\
\partial_{t} a_{1^{\prime}}=i c_{4} E_{S}^{*} a_{1} .
\end{array}\right.
$$

\section{Remark 2:}

We do not know how to improve the growth in time of our solutions because we do not have intrinsic bounds for the $\partial^{\alpha} U_{i}$. However, one can see that for $\left(\mathbf{S}^{\prime}\right)$ and $\left(\mathbf{M}^{\prime}\right)$, the structure of $F\left(\mathbf{U}_{\mathbf{2}}\right)$ implies the following energy equality:

$$
\partial_{t}\left\|\mathbf{U}_{\mathbf{1 , i}}(t)\right\|_{L^{2}}^{2}=\sum_{j} c_{j} \partial_{t}\left\|\mathbf{U}_{\mathbf{2}, \mathbf{j}}(t)\right\|_{L^{2}}^{2} \quad \text { where } c_{j} \geq 0
$$

This implies that $\mathbf{U}_{\mathbf{1}}(t)$ in $L^{\infty}\left(\mathbb{R}_{t}^{+} ; L_{x}^{2}\left(\mathbb{R}^{n}\right)\right)$.

\subsection{The case $\mathbb{R}^{3}$}

For a three dimensional geometry, the previous theorem is no longer valid for any arbitrary differential operator $i P(D)$. Indeed, the bounds on the $H^{1}$ norm are not sufficient in order to control $\left\|\partial_{x} \mathbf{U}_{\mathbf{1}} \partial_{x} \mathbf{U}_{\mathbf{2}}\right\|_{2}$ since the equivalent of the logarithmic 2D Sobolev inequality involves $\|\mathbf{U}\|_{H^{3 / 2}}$ instead of $\|\mathbf{U}\|_{H^{1}}$. Nevertheless, if we assume that $i P(D)$ is a Schrödinger-type operator, we can state the following result:

Theorem 3.2. Let us consider the following system:

$$
\left\{\begin{array}{l}
\partial_{t} \mathbf{U}_{\mathbf{1}}+i \triangle_{x} \mathbf{U}_{\mathbf{1}}=i F\left(\mathbf{U}_{\mathbf{2}}\right) \\
\partial_{t} \mathbf{U}_{\mathbf{2}}=i H \mathbf{U}_{\mathbf{2}}+A\left(\mathbf{U}_{\mathbf{1}}\right) \mathbf{U}_{\mathbf{2}}
\end{array}\right.
$$


with the same assumptions as above.

Then, for an initial Cauchy data in $H^{s}\left(\mathbb{R}^{3}\right)$ with $s>3 / 2$, the previous system has a unique solution $\mathbf{U}=\left(\mathbf{U}_{\mathbf{1}}, \mathbf{U}_{\mathbf{2}}\right)$ in $\mathcal{C}^{0}\left(\mathbb{R}_{t}^{+}, H^{s}\left(\mathbb{R}^{3}\right)\right) \cap \mathcal{C}^{1}\left(\mathbb{R}_{t}^{+}, H^{s-2}\left(\mathbb{R}^{3}\right)\right)$.

Moreover, one has $\mathbf{U}_{\mathbf{2}}$ in $L^{\infty}\left(\mathbb{R}_{t}^{+} ; L_{x}^{2}\left(\mathbb{R}^{3}\right)\right) \cap L^{\infty}\left(\mathbb{R}_{t}^{+} \times \mathbb{R}_{x}^{3}\right)$.

The same result occurs if we consider $\left(\partial_{x_{1}}+i \triangle_{x_{2}, x_{3}}\right)$ instead of $i \triangle_{x}$.

Proof. The same arguments as for the previous theorem give the following energy estimates:

$$
\left\|\mathbf{U}_{\mathbf{1 , 2}}\right\|_{L^{\infty}\left([0, t] ; H^{1}\right)} \leq C(t) ; \quad\left\|\mathbf{U}_{\mathbf{2}}\right\|_{L^{\infty}\left([0, t] \times \mathbb{R}^{3}\right)} \leq C(t) ;
$$

where $C(t)$ is $L_{\mathrm{loc}}^{\infty}\left(\mathbb{R}_{t}^{+}\right)$.

Using the relations (34), (36) of the previous theorem, we have (with the notation $\left.\|\mathbf{U}\|_{H^{2}}=\left\|\mathbf{U}_{\mathbf{1}}\right\|_{H^{2}}+\left\|\mathbf{U}_{\mathbf{2}}\right\|_{H^{2}}\right)$ :

$$
\|\mathbf{U}(t)\|_{H^{2}}^{2} \leq\left\|\mathbf{U}^{\mathbf{0}}\right\|_{H^{2}}^{2}+C_{0} \int_{0}^{t}\|\mathbf{U}(s)\|_{H^{2}}^{2} d s+C_{0} \int_{0}^{t}\left\|\partial_{x} \mathbf{U}_{\mathbf{1}}(s)\right\|_{L^{4}}^{2}\left\|\partial_{x} \mathbf{U}_{\mathbf{2}}(s)\right\|_{L^{4}}^{2} d s .
$$

Using an Hölder inequality, we have:

$$
\begin{aligned}
\|\mathbf{U}(t)\|_{H_{2}}^{2} \leq & \left\|\mathbf{U}^{\mathbf{0}}\right\|_{H^{2}}^{2}+C_{0} \int_{0}^{t}\|\mathbf{U}(s)\|_{H^{2}}^{2} d s+ \\
& C_{0}\left(\int_{0}^{t}\left\|\partial_{x} \mathbf{U}_{\mathbf{1}}(s)\right\|_{L^{4}}^{8 / 3} d s\right)^{3 / 4}\left(\int_{0}^{t}\left\|\partial_{x} \mathbf{U}_{\mathbf{2}}(s)\right\|_{L^{4}}^{8} d s\right)^{1 / 4} .
\end{aligned}
$$

But now, we can use the classical $L^{p}-L^{q}$ estimates on Schrödinger equation to obtain:

$$
\begin{aligned}
\left\|\partial_{x} \mathbf{U}_{\mathbf{1}}\right\|_{L^{8 / 3}\left(L^{4}\right)} & \leq C_{1}\left\|\partial_{x} \mathbf{U}_{\mathbf{1}}(0)\right\|_{L^{2}}+C_{1}\left\|\partial_{x} F\left(\mathbf{U}_{\mathbf{2}}\right)\right\|_{L^{\infty}\left(L^{2}\right)}, \\
& \leq C_{1}(1+C(t)),
\end{aligned}
$$

where $C_{1}$ depends on $\left\|\mathbf{U}_{1}^{0}\right\|_{H^{1}}$.

Using the Gagliardo-Niremberg estimate on $\left\|\partial_{x} \mathbf{U}_{\mathbf{2}}\right\|_{4}$, we obtain:

$$
\begin{aligned}
\|\mathbf{U}(t)\|_{H^{2}}^{2} & \leq\left\|\mathbf{U}^{0}\right\|_{H^{2}}^{2}+C_{0} \int_{0}^{t}\|\mathbf{U}(s)\|_{H^{2}}^{2} d s+\widetilde{C}(t)\left(\int_{0}^{t}\left\|\partial_{x} \mathbf{U}_{\mathbf{2}}(s)\right\|_{L^{4}}^{8} d s\right)^{1 / 4} \\
& \leq\left\|\mathbf{U}^{0}\right\|_{H^{2}}^{2}+C_{0} \int_{0}^{t}\|\mathbf{U}(s)\|_{H^{2}}^{2} d s+\widetilde{C}(t) \int_{0}^{t}\left\|\mathbf{U}_{\mathbf{2}}(s)\right\|_{H^{2}} d s
\end{aligned}
$$

The conclusion follows with a Gronwall's lemma.

Now, one can wonder if the previous theorem is still valid for the Maxwell operator, i.e for the system:

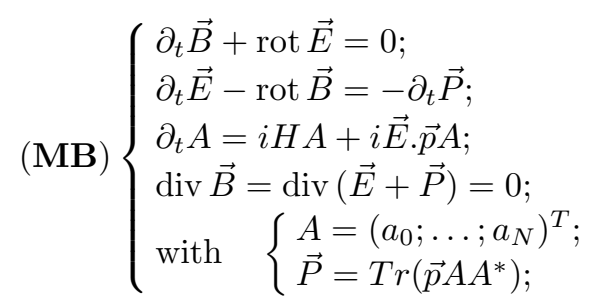


where $\vec{p}$ is the hermitian (complex) matrix of the electronic polarizations.

The answer is yes but it is important to understand that the proof of this result is intimately linked with the properties of the electronic polarizations $\vec{p}_{i j}$ which couple Maxwell and Bloch equations. Indeed, denoting by $\rho$ the matrix $A A^{*}$, we have:

$$
\partial_{t} \vec{P}=\operatorname{Tr}(\vec{p}[i H ; \rho])+\operatorname{Tr}(i \vec{E} \cdot[\vec{p} ; \rho]) \quad \text { where } \quad[A ; B]=A B-B A .
$$

So, the $k^{\text {th }}$ component of this vector has the form:

$$
\partial_{t} P^{k}=\operatorname{Tr}\left(p^{k}[i H ; \rho]\right)+i \sum_{j=1}^{3} E^{j} \operatorname{Tr}\left(\left[p^{k} ; p^{j}\right] \rho\right) .
$$

But now, it is clear that the second term on the r.h.s of (37) has no reason to vanish without assumptions on the polarizations. A sufficient (but not necessary) condition to ensure the cancellation of this term is $\vec{p}_{i j}=p_{i j} \vec{u}$ with $p_{i j} \in \mathbb{C}$ and $\vec{u} \in \mathbb{R}^{3}$. Such an assumption is often implicitly used in the literature. For example, in many texts[4], [8]' [15], the two-level Maxwell-Bloch system is expressed in the form:

$$
(\mathbf{D R})\left\{\begin{array}{l}
\partial_{t} \vec{B}+\operatorname{rot} \vec{E}=0 ; \\
\partial_{t} \vec{E}-\operatorname{rot} \vec{B}=-\partial_{t} \vec{P} ; \\
\partial_{t^{2}}^{2} \vec{P}+\partial_{t} \vec{P}+\vec{P}=N \vec{E} ; \\
\partial_{t} N+N=<\partial_{t} \vec{P} ; \vec{E}>
\end{array}\right.
$$

Here we claim that this system is only valid if the electronic polarization $\vec{p}_{01}$ is a real vector or has the form $\vec{p}_{01}=\alpha \vec{u}$ with $\alpha \in \mathbb{C}$ and $\vec{u} \in \mathbb{R}^{3}$.

For such Maxwell-Bloch systems, the existence of global solutions was proved by Donnat and Rauch[8]. The key point is that the cancellation of the second term in the r.h.s of (37) gives a control of the $L^{\infty}$ norm of the "source term" $\partial_{t} \vec{P}$ in the Maxwell part of the system.

For general Maxwell-Bloch systems, $\partial_{t} \vec{P}$ involves terms in $\vec{E} a_{i} a_{j}^{*}$ and the analysis of Donnat-Rauch is no longer valid. Fortunately, we can use the work of Joly-Métivier-Rauch[12] to ensure the result (see also the work of E.Dumas[9]). Indeed, in this paper, the authors deal with the following system:

$$
(\mathbf{J M R})\left\{\begin{array}{l}
\partial_{t} \vec{E}-\operatorname{rot} \vec{H}=0 \\
\partial_{t} \vec{H}+\operatorname{rot} \vec{E}=-\partial_{t} \vec{M} \\
\partial_{t} \vec{M}=F(\vec{M}, \vec{H}) .
\end{array}\right.
$$

Assuming the function $F$ satisfies

$$
\begin{cases}h \longmapsto F(m, h) & \text { is linear } \forall m \in \mathbb{R}^{3} \\ F(m, h) . m=0 & \forall(m, h) \in \mathbb{R}^{3}\end{cases}
$$


the authors prove that $\|(\vec{E}, \vec{H}, \vec{M})(t)\|_{H^{s}} \leq C e^{C^{\prime} e^{t}}$ for all $s \geq 0$ (the key points are an Hodge decomposition, limit Strichartz-type estimates and time derivatives estimates).

This analysis can be directly applied to the system (MB) since the interaction between Maxwell and Bloch equations are only linear in term of $\vec{E}$ and since the fundamental law $R e<\vec{E} \cdot \vec{p} A, A>=0$ plays the rule of $F(m, h) \cdot m=0$.

Finally, this allows us to state the following fundamental result:

\section{Theorem 3.3. (Joly-Métivier-Rauch-Dumas)}

Let us consider the system:

$$
(\mathbf{M B})\left\{\begin{array}{l}
\partial_{t} \vec{B}+\operatorname{rot} \vec{E}=0 \\
\partial_{t} \vec{E}-\operatorname{rot} \vec{B}=-\partial_{t} \vec{P} \\
\partial_{t} A=i H A+i \vec{E} \cdot \vec{p} A \\
\operatorname{div} \vec{B}=\operatorname{div}(\vec{E}+\vec{P})=0 \\
A=\left(a_{0} ; \ldots ; a_{N}\right)^{T} \\
\vec{P}=\operatorname{Tr}\left(\vec{p} A A^{*}\right)
\end{array}\right.
$$

where $H \in \mathcal{M}^{N+1}(\mathbb{C})$ and $\vec{p}$ is an hermitian matrix.

Then, for an initial Cauchy data $U_{0}$ in $H^{s}\left(\mathbb{R}^{3}\right)$ with $s>3 / 2$ such that div $\vec{B}_{0}=$ $\operatorname{div}\left(\vec{E}_{0}+\vec{P}_{0}\right)=0$, the previous system has a unique solution in $\mathcal{C}^{0}\left(\mathbb{R}_{t}^{+}, H^{s}\left(\mathbb{R}^{3}\right)\right) \cap$ $\mathcal{C}^{1}\left(\mathbb{R}_{t}^{+}, H^{s-1}\left(\mathbb{R}^{3}\right)\right)$.

Moreover, one has $(\vec{B} ; \vec{E} ; A)$ in $L^{\infty}\left(\mathbb{R}_{t}^{+} ; L_{x}^{2}\left(\mathbb{R}^{3}\right)\right)$ and $A$ in $L^{\infty}\left(\mathbb{R}_{t}^{+} \times \mathbb{R}_{x}^{3}\right)$.

\section{Remark 3:}

The work of Joly-Métivier-Rauch contains in fact many others useful informations. Mainly, it can be used to build solutions $\left(\vec{B}, \vec{E}, a_{0}, \ldots, a_{N}\right)$ of $(\mathbf{M B})$ in $\left(H^{s}\right)^{2} \times\left(H^{s} \cap L^{\infty}\right)^{N+1}$ with $s \geq 0$ (nevertheless for $s<1$ we do not have the uniqueness). Such a study has been recently done by E.Dumas[9].

\section{A discussion of Raman amplification}

In this section, we give a brief discussion of Raman amplification.

The resonance assumption $\omega_{L}-\omega_{S}=\omega_{1}$ leads to a growth of the Raman component $E_{S}$ of the electric field. Such a behavior has already been studied by many people and there is no optics textbook without an analysis (at least in a formal way) of this phenomena (see Boyd and Newell[4]'[15]). One of the main conclusions of these studies is related to the following formula:

$$
I_{S}=c e^{g x I_{L}}
$$

where $I_{L}$ and $I_{S}$ denote the intensity of the laser pulse and of the Raman field, $(g, c)$ denote constants related to the physical characteristics of the initial pulse 
and of the medium and $x$ is the axis of propagation.

This formula is really useful since it is in agreement with many physical experiments. Nevertheless, it is obtained after many approximations (the higher energy level is often dropped, the structure of the non-linearities is simplified ...) which are not studied. Such justifications seem difficult and, as far as we are concerned, we do not have any interesting arguments.

In this paragraph, we just show that Raman amplification is a phenomena of instability.

Our discussion is based on the 1D version of our system:

$$
\left(\mathbf{M}^{\prime \prime}\right)\left\{\begin{array}{l}
\left(\partial_{t}+\partial_{x}\right) E_{L}=i c_{1} a_{1} a_{0}^{*} \\
\left(\partial_{t}+\partial_{x}\right) E_{S}=i c_{2} a_{1} a_{1^{\prime}}^{*} \\
\partial_{t} a_{0}=i c_{3} E_{L}^{*} a_{1} \\
\partial_{t} a_{1}=-i a_{1} / \eta+i c_{3} E_{L} a_{0}+i c_{4} E_{S} a_{1^{\prime}} \\
\partial_{t} a_{1^{\prime}}=i c_{4} E_{S}^{*} a_{1} .
\end{array}\right.
$$

It is still difficult to give a qualitative study of $\left(\mathbf{M}^{\prime \prime}\right)$ without additional simplifications. First, it is convenient to consider only the resonant case $1 / \eta=0$ since it allows us to work with real-valued variables. Indeed, if the Cauchy data verifies $\left(E_{L}^{0} ; E_{S}^{0} ; a_{0}^{0} ; a_{1^{\prime}}^{0}\right) \in \mathbb{R}$ and $a_{1}^{0} \in i \mathbb{R}$, then the solution stays in this space and, changing $a_{1}$ into $i a_{1}$, the system takes the form:

$$
\left(\mathbf{M}_{\mathbf{r}}^{\prime \prime}\right)\left\{\begin{array}{l}
\left(\partial_{t}+\partial_{x}\right) E_{L}=-c_{1} a_{1} a_{0} \\
\left(\partial_{t}+\partial_{x}\right) E_{S}=-c_{2} a_{1} a_{1^{\prime}} \\
\partial_{t} a_{0}=-c_{3} E_{L} a_{1} \\
\partial_{t} a_{1}=c_{3} E_{L} a_{0}+c_{4} E_{S} a_{1^{\prime}} \\
\partial_{t} a_{1^{\prime}}=-c_{4} E_{S} a_{1}
\end{array}\right.
$$

Now, since the group associated with the transport operator $\partial_{t}+\partial_{x}$ is bounded for the $L^{\infty}$ norm, we can construct solutions of ( $\left.\mathbf{M}_{\mathbf{r}}^{\prime \prime}\right)$ for Cauchy data's in $L^{\infty}$. Taking an initial data independent of $x$, we can drop the $\partial_{x}$ and discuss the dynamic of the following system of ordinary differential equations:

$$
(\widetilde{\mathbf{M}})\left\{\begin{array}{l}
\partial_{t} E_{L}=-c_{1} a_{1} a_{0} \\
\partial_{t} E_{S}=-c_{2} a_{1} a_{1^{\prime}} \\
\partial_{t} a_{0}=-c_{3} E_{L} a_{1} \\
\partial_{t} a_{1}=c_{3} E_{L} a_{0}+c_{4} E_{S} a_{1^{\prime}} \\
\partial_{t} a_{1^{\prime}}=-c_{4} E_{S} a_{1} .
\end{array}\right.
$$

Before going further, let us discuss the validity of these simplifications. First, the consideration of the resonant case seems interesting since it is clear that the speed of the Raman amplification is maximal in this case. In fact, we claim that the factor $\eta$ decreases (however we do not know in which way yet) the speed of the energy transfer between $E_{L}$ and $E_{S}$ but do not influence the intensity 
of this transfer. To neglect the spatial derivatives seems to be a more severe simplification but it can be seen as the first step of a local analysis.

Now, we return to the study of $(\widetilde{\mathbf{M}})$. We are concerned with a Cauchy data $\left(E_{L}^{0} ; \epsilon E_{S}^{0} ; 1 ; 0 ; 0\right)$ where $\epsilon \ll 1$ and $\left(E_{L}^{0} ; E_{S}^{0}\right) \sim \mathcal{O}(1)$. The cornerstone of our discussion is that for $\epsilon=0$, the solution of $(\widetilde{\mathbf{M}})$ is $\left(E_{L} ; 0 ; a_{0} ; a_{1} ; 0\right)$ where $\left(E_{L} ; a_{0} ; a_{1}\right)$ is the solution of the two-level system:

$$
\left(\widetilde{\mathbf{M}_{1}}\right)\left\{\begin{array}{l}
\partial_{t} E_{L}=-c_{1} a_{1} a_{0} \\
\partial_{t} a_{0}=-c_{3} E_{L} a_{1} \\
\partial_{t} a_{1}=c_{3} E_{L} a_{0}
\end{array}\right.
$$

We show in the following proposition that $\left(E_{S} ; a_{1^{\prime}}\right)=(0 ; 0)$ is an unstable equilibrium for $(\widetilde{\mathbf{M}})$.

\section{Proposition 4.1.}

For the system $(\widetilde{\mathbf{M}})$, the dynamic equilibrium $\left(E_{L}(t) ; E_{S}=0 ; a_{0}(t) ; a_{1}(t) ; a_{1^{\prime}}=\right.$ 0) is unstable, i.e:

$\exists \epsilon>0$ and $t^{\epsilon}>0 / \forall \rho>0, \forall\left(E_{S}^{0}, a_{1^{\prime}}^{0}\right),,\left|E_{S}(0) ; a_{1^{\prime}}(0)\right| \leq \rho \Longrightarrow\left|E_{S}\left(t^{\epsilon}\right) ; a_{1^{\prime}}\left(t^{\epsilon}\right)\right|>\epsilon$.

Proof. Suppose the equilibrium is stable. This means that

$$
\forall \epsilon>0, \quad \exists \rho^{\epsilon}>0 \quad / \forall\left(E_{S}^{0}, a_{1^{\prime}}^{0}\right),\left|E_{S}^{0} ; a_{1^{\prime}}^{0}\right| \leq \rho \Longrightarrow\left|E_{S}(t) ; a_{1^{\prime}}(t)\right| \leq \epsilon, \quad \forall t \geq 0 .
$$

Plugging this assumption into $(\widetilde{\mathbf{M}})$ leads to:

$$
(\widetilde{\mathbf{M}})\left\{\begin{array}{l}
\partial_{t} E_{L}=-c_{1} a_{1} a_{0} \\
\partial_{t} E_{S}=-c_{2} a_{1} a_{1^{\prime}} \\
\partial_{t} a_{0}=-c_{3} E_{L} a_{1} \\
\partial_{t} a_{1}=c_{3} E_{L} a_{0}+\epsilon^{2} c_{4} E_{S} a_{1^{\prime}}=c_{3} E_{L} a_{0}+\mathcal{O}\left(\epsilon^{2}\right) \\
\partial_{t} a_{1^{\prime}}=-c_{4} E_{S} a_{1} .
\end{array}\right.
$$

So, at order $\mathcal{O}(1)$, the assumption splits the system $(\widetilde{\mathbf{M}})$ into:

$$
\left(\widetilde{\mathbf{M}_{1}}\right)\left\{\begin{array} { l } 
{ \partial _ { t } E _ { L } = - c _ { 1 } a _ { 1 } a _ { 0 } , } \\
{ \partial _ { t } a _ { 0 } = - c _ { 3 } E _ { L } a _ { 1 } , } \\
{ \partial _ { t } a _ { 1 } = c _ { 3 } E _ { L } a _ { 0 } , }
\end{array} \quad ( \widetilde { \mathbf { M } _ { 2 } } ) \left\{\begin{array}{l}
\partial_{t} E_{S}=-c_{2} a_{1} a_{1^{\prime}} \\
\partial_{t} a_{1^{\prime}}=-c_{4} E_{S} a_{1} .
\end{array}\right.\right.
$$

Now, since the time-dependent matrix

$$
\left(\begin{array}{ll}
0 & -c_{2} a_{1}(t) \\
-c_{4} a_{1}(t) & 0
\end{array}\right)
$$


commutes for all $\mathrm{t}$ (this is the reason why we have considered the resonant case), we have:

$$
\left\{\begin{array}{l}
E_{S}(t)=E_{S}^{0} \operatorname{ch}\left(\sqrt{c_{2} c_{4}} \int_{0}^{t} a_{1}(s) d s\right), \\
a_{1^{\prime}}(t)=-\sqrt{\frac{c_{4}}{c_{2}}} E_{S}^{0} \operatorname{sh}\left(\sqrt{c_{2} c_{4}} \int_{0}^{t} a_{1}(s) d s\right) .
\end{array}\right.
$$

So, in order to obtain the suitable contradiction, it is sufficient to prove that $a_{1}$ has a limit $l \neq 0$ when $t \longrightarrow+\infty$ or that $a_{1}$ is periodic.

Consequently, we study the system $\left(\widetilde{\mathbf{M}_{1}}\right)$ with respect to the Cauchy data $\left(E_{L}^{0} ; 1 ; 0\right)$ (of course, we take $E_{L}^{0} \neq 0$ ).

First, change $E_{L}$ into $\sqrt{c_{3} / c_{1}} E_{L}$ to obtain the homogeneous system:

$$
\left(\widetilde{\mathbf{M}_{\mathbf{1 1}}}\right)\left\{\begin{array}{l}
\partial_{t} E_{L}=-c a_{1} a_{0}, \\
\partial_{t} a_{0}=-c E_{L} a_{1}, \\
\partial_{t} a_{1}=c E_{L} a_{0},
\end{array}\right.
$$

where $c=\sqrt{c_{1} c_{3}}$.

The first two equations lead to:

$$
\left(E_{L}-a_{0}\right)(t)=e^{c \int_{0}^{t} a_{1}(s) d s}\left(E_{L}^{0}-a_{0}^{0}\right)
$$

So, if the Cauchy data satisfies $E_{L}^{0}=a_{0}^{0}=1$, we have $E_{L}=a_{0}$. Now, recall that we have the fundamental energy estimates:

$$
\left\{\begin{array}{l}
\left|E_{L}\right|^{2}=\left|E_{L}^{0}\right|^{2}+\left|a_{0}\right|^{2}-\left|a_{0}^{0}\right|^{2} \\
\left|a_{0}\right|^{2}+\left|a_{1}\right|^{2}=\left|a_{0}^{0}\right|^{2}+\left|a_{1}^{0}\right|^{2}=1
\end{array}\right.
$$

Hence, we can solve the system $\left(\widetilde{\mathbf{M}_{11}}\right)$ as:

$$
\left\{\begin{array}{l}
a_{1}(t)=t h(c t) \\
a_{0}(t)=E_{L}(t)=1 / \operatorname{ch}(c t)
\end{array}\right.
$$

The case $E_{L}^{0}=-a_{0}^{0}=-1$ is similar and leads to:

$$
\left\{\begin{array}{l}
a_{1}(t)=t h(-c t), \\
a_{0}(t)=-E_{L}(t)=1 / \operatorname{ch}(c t) .
\end{array}\right.
$$

Thus, for $\left|E_{L}^{0}\right|=\left|a_{0}^{0}\right|$, this proves the instability of the equilibrium $\left(E_{S} ; a_{1^{\prime}}\right)=$ $(0 ; 0)$.

Now, we will see that for $\left|E_{L}^{0}\right| \neq\left|a_{0}^{0}\right|$, the system $\left(\widetilde{\mathbf{M}_{\mathbf{1 1}}}\right)$ has a periodic solution. For this, the key point is to remark that $\left(\widetilde{\mathbf{M}_{11}}\right)$ has only two equilibriums $\left(0 ; 0 ; a_{1}^{0}\right)$ and $\left(0 ; a_{0}^{0} ; 0\right)$ for a Cauchy data such that $\left|a_{0}^{0}\right|^{2}+\left|a_{1}^{0}\right|^{2}>0$. Using the energy estimates, we see that for a Cauchy data $\left(E_{L}^{0} ; 1 ; 0\right)$ such that $E_{L}^{0} \neq 0$ and $\left|E_{L}^{0}\right| \neq\left|a_{0}^{0}\right|$, any component of the solution can be constant on an interval ]$t_{0} ; t_{1}[$ or be directed toward one of the previous equilibriums when $t \longrightarrow+\infty$. 
Using the boundedness of the solution, this behavior implies the periodicity of the solution. Indeed, let us suppose that $E_{L}^{0}>a_{0}^{0}=1$ (in fact we could treat any Cauchy data). The equation for $\left(E_{L}-a_{0}\right)$ shows that $E_{L}(t)>a_{0}(t)$ and the energy estimates show that $E_{L}(t)>0$ for all $t$. But now, since the Cauchy data $\left(E_{L}^{0}, a_{0}^{0}, 0\right)$ is not an equilibrium, since $E_{L}(t) \leq E_{L}^{0}$ and $a_{0}(t) \leq 1$, since $\partial_{t} a_{1}(0)=c E_{L}(0)>0$ and since there is no stationary cycle on an interval ]$t_{1} ; t_{2}[$, it makes sense to define:

$t^{1}=\sup \left\{t>0\right.$ such that $\partial_{t} E_{L}\left(t^{\prime}\right)<0$ and $\partial_{t} a_{0}\left(t^{\prime}\right)<0$ and $\left.\partial_{t} a_{1}\left(t^{\prime}\right)>0, \forall t^{\prime} \in\right] 0 ; t[\}$.

Now, since the derivative of any variable cannot be directed toward 0 when $t \longrightarrow+\infty$ and since the solution is bounded, we have $t^{1}<+\infty$ and so the derivate of one variable must vanish at $t^{1}$. It is easy to see that it implies $a_{0}\left(t^{1}\right)=0, a_{1}\left(t^{1}\right)=1$ and $E_{L}\left(t^{1}\right)=\sqrt{\left|E_{L}^{0}\right|^{2}-\left|a_{0}^{0}\right|^{2}}$. So, we have:

$$
\left\{\begin{array} { l } 
{ \partial _ { t } E _ { L } ( t ^ { 1 } ) = 0 ; } \\
{ \partial _ { t } a _ { 1 } ( t ^ { 1 } ) = 0 ; } \\
{ \partial _ { t } a _ { 0 } ( t ^ { 1 } ) = - c E _ { L } ( t ^ { 1 } ) < 0 ; }
\end{array} \quad \left\{\begin{array}{l}
\partial_{t}^{2} E_{L}\left(t^{1}\right)=c^{2} E_{L}\left(t^{1}\right)>0 ; \\
\partial_{t}^{2} a_{1}\left(t^{1}\right)=-c^{2} E_{L}^{2}\left(t^{1}\right)<0 ; \\
\partial_{t}^{2} a_{0}\left(t^{1}\right)=0 .
\end{array}\right.\right.
$$

But now, it is clear that these relations imply that the same discussion can be made on an interval $] t^{1} ; t^{2}$ [ and will lead to the state $\left(E_{L}\left(t^{2}\right) ; a_{0}\left(t^{2}\right) ; a_{1}\left(t^{2}\right)\right)=$ $\left(E_{L}^{0} ;-1 ; 0\right)$. In the same spirit, we can find a $t^{3}$ such that $\left(E_{L}\left(t^{3}\right) ; a_{0}\left(t^{3}\right) ; a_{1}\left(t^{3}\right)\right)=$ $\left(E_{L}\left(t^{1}\right) ; 0 ;-1\right)$ and a $t^{4}$ such that $\left(E_{L}\left(t^{4}\right) ; a_{0}\left(t^{4}\right) ; a_{1}\left(t^{4}\right)\right)=\left(E_{L}^{0} ; 1 ; 0\right)$.

Since we have already noticed that this discussion is possible for any Cauchy data, this proves our assertion on the periodicity of the solutions and on the instability of the equilibrium $\left(E_{S} ; a_{1^{\prime}}\right)=(0 ; 0)$.

\section{Comment:}

Throughout a simple example, we tried to explain why the three-level MaxwellBloch system could generate a secular growth of the Raman component of the electric field. This study is far from complete. It will be interesting to overcome our simplifications (on $\eta$ and $\partial_{x}$ ). We think that the qualitative analysis of the system $\left(\widetilde{\mathbf{M}_{\mathbf{1 1}}}\right)$ could be extended to the initial system $(\widetilde{\mathbf{M}})$. There are at least two major points to study:

- the search of particular solutions in terms of hyperbolic functions. It is of fundamental interest since the apparition of $t h(c t)$ could explain both the amplification regime (instead of the formula (38)) and the important saturation regime (up to now, there are no mathematical qualitative studies of this phenomenon excepted in the area of the Inverse Scattering Transform - see Kaup and al.[13] and its bibliography).

- the existence of periodic or quasi-periodic solutions. 


\section{A numerical illustration of Raman amplifica- tion}

In the previous paragraph, we saw that the general Maxwell-Bloch system $(\mathbf{S})$ is approximated by the reduced system $(\mathbf{M})$ on an interval $[0 ; T]$ with an error in $C(T) \sqrt{\epsilon}$. It is of fundamental interest for practical applications to have a precise estimate on $C(T)$ and on the error in terms of the parameters. This is done by numerical computations.

The design of efficient schemes for $(\mathbf{M})$ is a difficult task because one must take into account different sources of stiffness: the growth of $E_{S}$ and oscillations generated by $e^{i t / \eta}$ for $Q_{1}$ and $Q_{3}$. Moreover, spatial oscillations may appear for long times and/or in the saturation regime (i.e when the growth of $E_{S}$ stops). We postpone to a further work the study of these regimes and restrict to a simple numerical illustration of Raman amplification.

We consider the following regime in the $1 \mathrm{D}$ case:

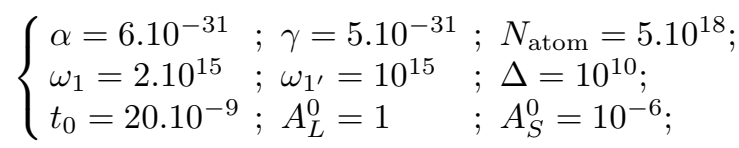

where $A_{L, S}^{0}$ are related to the Cauchy data by

$$
\left(\begin{array}{l}
E_{L}(t=0) \\
E_{S}(t=0) \\
a_{0}(t=0) \\
a_{1}(t=0) \\
a_{1^{\prime}}(t=0)
\end{array}\right)=\left(\begin{array}{l}
A_{L}^{0} e^{-\ln (\sqrt{2}) x^{2}} \\
A_{S}^{0} e^{-\ln (\sqrt{2}) x^{2}} \\
1 \\
0 \\
0
\end{array}\right)
$$

For this regime, the asymptotic parameters are $\epsilon=2.5 * 10^{-8}$ and $\eta=5.10^{-3}$.

For these computations, we used a splitting approach. With Bloch variables, we can write $(\mathbf{M})$ as

$$
\left\{\begin{array}{l}
\partial_{t} \mathbf{U}_{\mathbf{1}}+\partial_{x} \mathbf{U}_{\mathbf{1}}=i H_{0} \mathbf{U}_{\mathbf{2}} \\
\partial_{t} \mathbf{U}_{\mathbf{2}}=i H_{1} \mathbf{U}_{\mathbf{1}}+i H_{2} \mathbf{U}_{\mathbf{2}}+A\left(\mathbf{U}_{\mathbf{1}}\right) \mathbf{U}_{\mathbf{2}}
\end{array}\right.
$$

where $\mathbf{U}_{1}=\left(E_{L} ; E_{S}\right) ; \mathbf{U}_{2}=\left(Q_{1} ; Q_{2} ; Q_{3}, N_{1} ; N_{2}\right)$ and $H_{i}$ denotes a real diagonal matrix related to the parameters of the system.

The numerical resolution is achieved in three steps:

\section{- Propagation step:}

we solve the transport equations on $\left(E_{L} ; E_{S}\right)$ using the method of characteristic lines. It is well-known that this method is exact and involves a classical CFL $\triangle t / \triangle x \leq 1$. For our test case, the time step is chosen in order to give a Courant number of unity and the computations are restricted to a moving window (at unit speed) related to the support of the initial pulses. 


\section{- Linear interaction:}

we solve analytically

$$
\left\{\begin{array}{l}
\partial_{t} \mathbf{U}_{\mathbf{1}}=i H_{0} \mathbf{U}_{\mathbf{2}} \\
\partial_{t} \mathbf{U}_{\mathbf{2}}=i H_{1} \mathbf{U}_{\mathbf{1}}+i H_{2} \mathbf{U}_{\mathbf{2}} .
\end{array}\right.
$$

\section{- Nonlinear interaction:}

we solve analytically (since $\mathbf{U}_{1}$ is fixed) the interaction Hamiltonian

$$
\partial_{t} \mathbf{U}_{\mathbf{2}}=A\left(\mathbf{U}_{\mathbf{1}}\right) \mathbf{U}_{\mathbf{2}}
$$

Now, let us consider the following computations ( for which the nondimensionnal numerical steps are $\triangle t=\triangle x=2 \cdot 10^{-3}$ ):

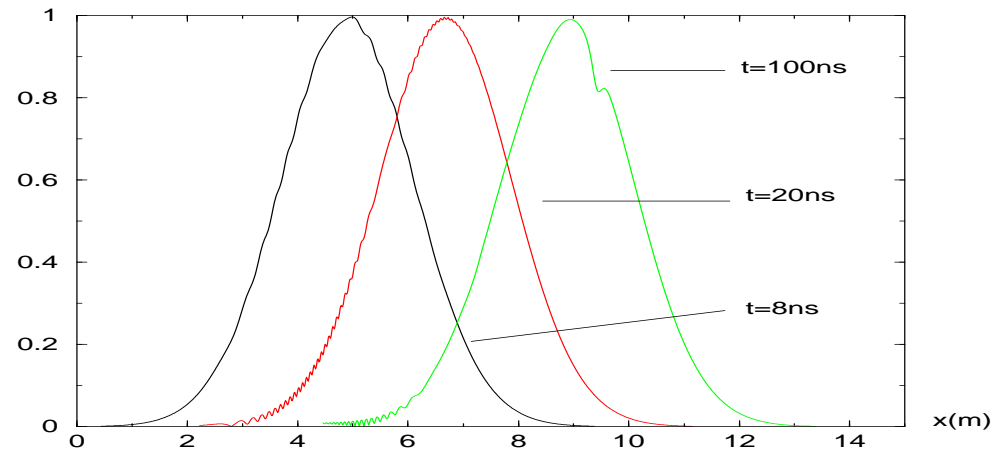

Fig. 1: transport of $\left|E_{L}\right| /\left|A_{L}^{0}\right|$

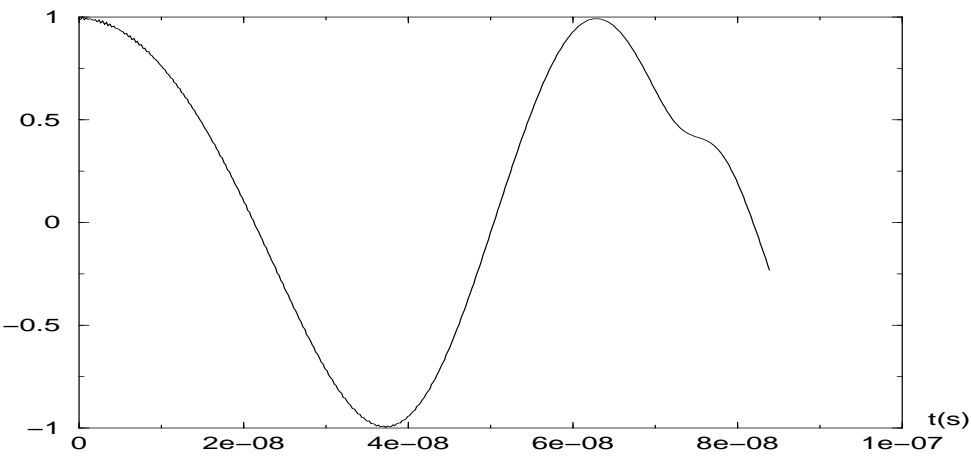

Fig. 2: rotation of $\operatorname{Re}\left(E_{L}\right) /\left|A_{L}^{0}\right|$

The transport of the amplitude of the fundamental laser pulse with a unit speed appears clearly at the beginning of the evolution like the rotation of its real component. Those behaviors, which occur before the Raman amplification, can be easily explained since they correspond to the evolution of a single pulse in the two-level atoms model. Therefore, the qualitative study of Colin-Nkonga[6] can be applied. 
More interesting is the regime of saturation that occurs during the Raman amplification (say after $10^{-7} s$ ). We see that this instability leads to a degradation of the Gaussian structure of the laser pulse. In the same way, the rotation of its real component is damaged.

Figures 3 and 4 are related to the amplification of the Raman component.

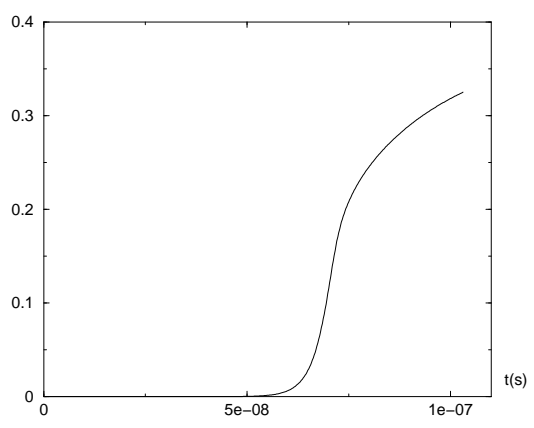

Fig. 3: amplification of $E_{S}$

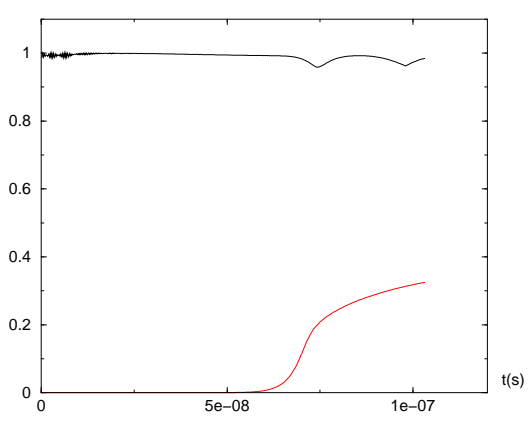

Fig. 4: maximum of $\left|E_{L}\right|$ and $\left|E_{S}\right|$

Figures 5-8 are related to the shape of the Raman component.

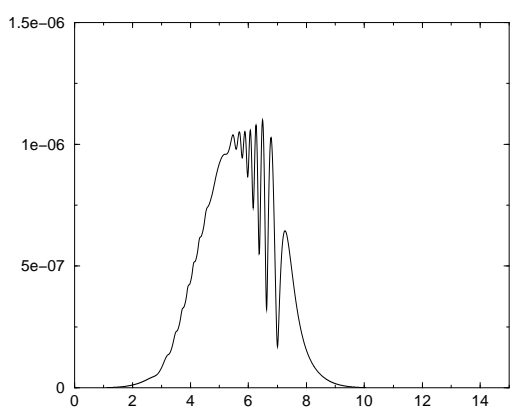

Fig. 5: $\left|E_{S}\right|^{2}$ at $t=10 n s$

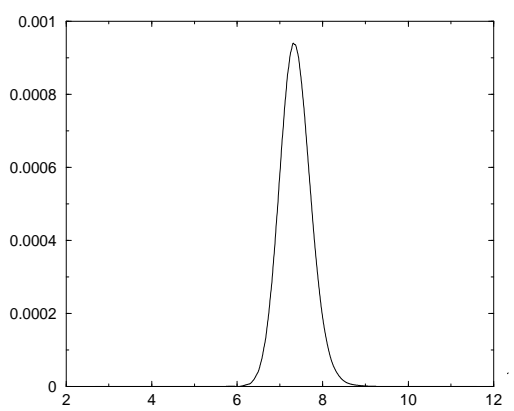

Fig. 7: $\left|E_{S}\right|^{2}$ at $t=35 n s$

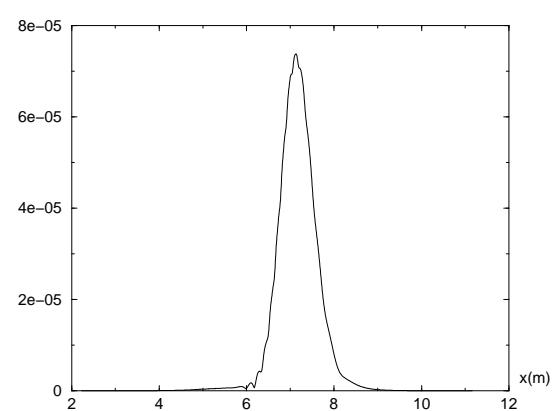

Fig. 6: $\left|E_{S}\right|^{2}$ at $t=20 n s$

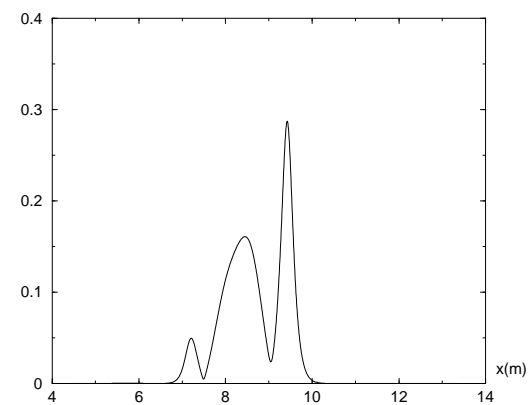

Fig. 8: $\left|E_{S}\right|^{2}$ at $t=100 n s$

Here the transport of the Raman component and its amplification appear clearly. The key point is the exponential feature in which this amplification occurs. We 
must also emphasize the fact that this instability generates spatial oscillations on the shape of the Raman pulse (up to now, we do not have suitable argument to explain this behavior).

We see that the Raman scattering is here efficient since after nearly $10^{-7} s$ the maximum of $\left|E_{S}\right|$ reaches forty percent of the amplitude of the initial laser pulse.

Now, let us consider the quantic coherences $Q_{i}$ at the time $t=10 \mathrm{~ns} ; t=20 \mathrm{~ns}$; $t=100 \mathrm{~ns}$.
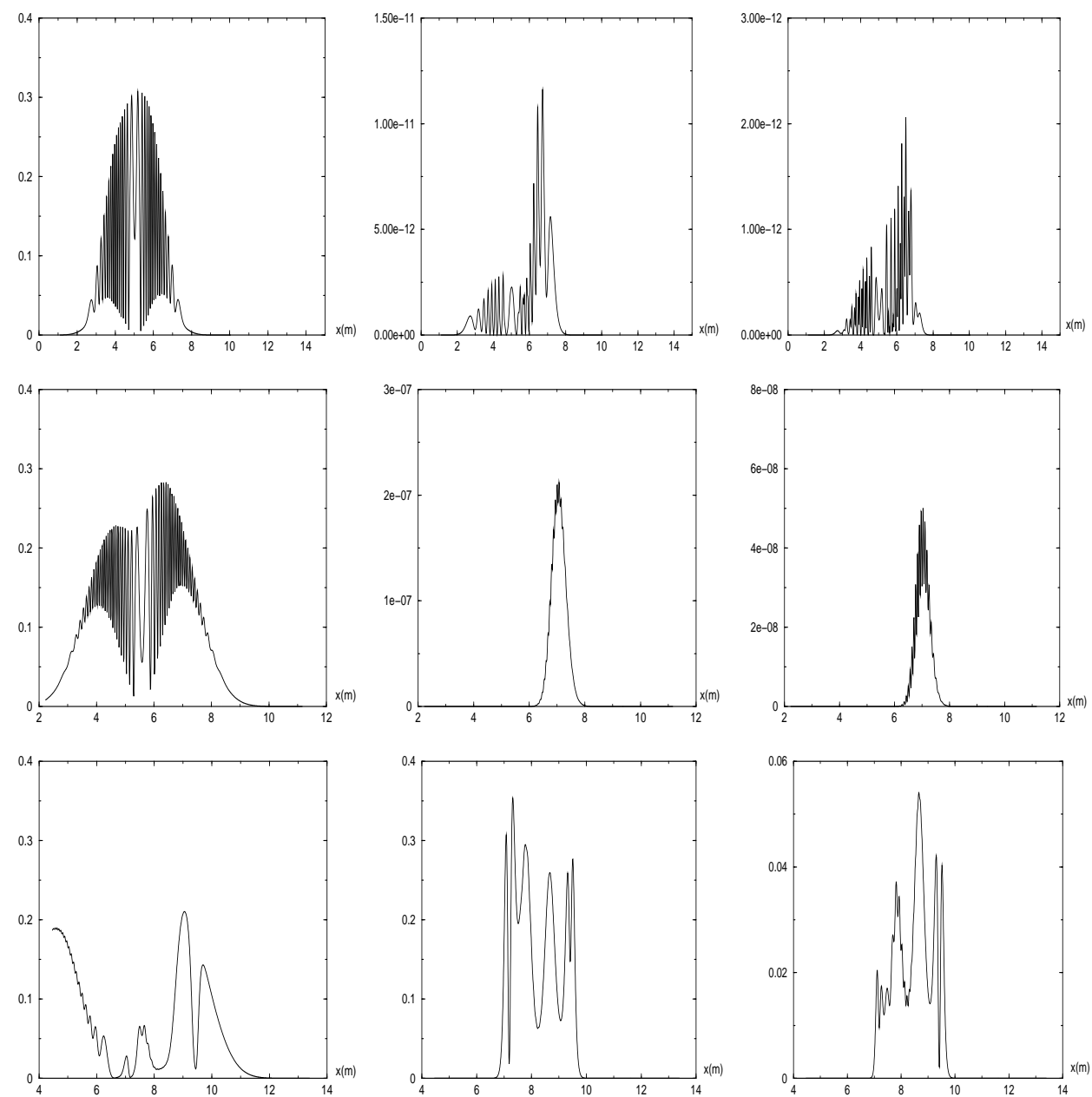

Evolution of $\left|Q_{1}\right|$

Evolution of $\left|Q_{2}\right|$

Evolution of $\left|Q_{3}\right|$

There are two important behaviors. The first one is the spatial oscillatory regime that occurs and which is clearly related to the behavior of $E_{S}$. The second one is the (expected) link between the maximums of $\left|Q_{1}\right|$ and $\left|E_{L}\right|$ on one side and of $\left|Q_{3}\right|$ and $\left|E_{S}\right|$ on the other side. 
This link between the quantic variables and the amplitudes of our pulses appears more clearly in the following pictures:

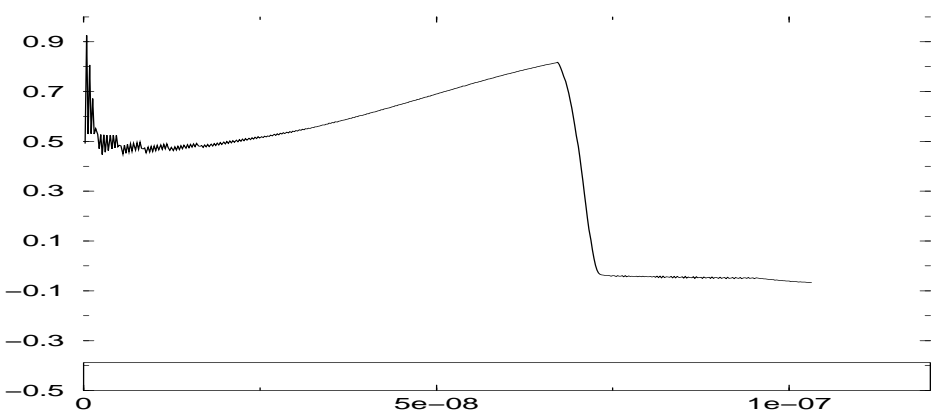

Fig. 8: minimum of $\left|a_{0}\right|^{2}$

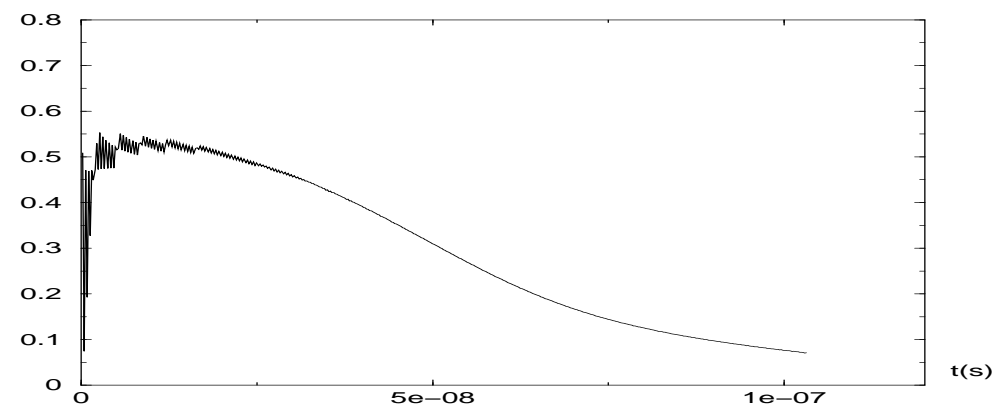

Fig. 9: maximum of $\left|a_{1}\right|^{2}$

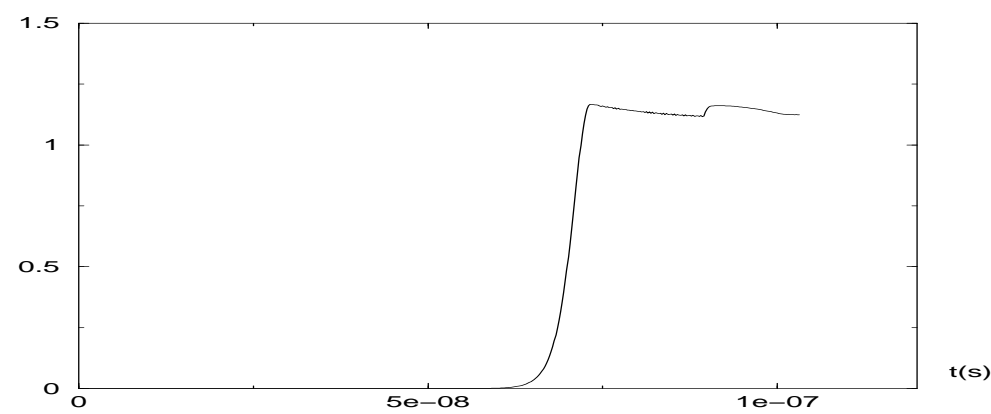

Fig. 10: maximum of $\left|a_{1^{\prime}}\right|^{2}$

Through these figures, we see that our scheme is still valid when inversion population arises (it is the case if the Raman amplification is sufficiently intense) but breaks down during the computation of the regime of saturation. Indeed, after $75 \mathrm{~ns}$, one can see that our scheme do not respect the fundamental conservation law for the quantic variables.

\section{Comment:}


In order to conclude, we can say that our asymptotic model (M) preserves the structure of the Raman amplification. However, these computations do not explain the important phenomenon of saturation which must control the behavior of $E_{S}$ after its amplification. For example, it is of fundamental interest to known if the spatial degradation of the shape of $E_{L}$ is time-reversible. In fact, as mentioned above, the numerical computation of this saturation is really hard and we postpone its study to a further work.

\section{Conclusion}

Dealing with a general version of three-level Maxwell-Bloch system, we have focused our attention on the study of stimulated Raman scattering in a gas medium. The scaling of the equations allowed to perform a rigorous asymptotic derivation of the initial model and led to Schrödinger equations for both the fundamental laser pulse and the Raman component. Through a numerical example, we have shown that this asymptotic approach preserves the qualitative aspect of Raman scattering. As mentioned in Colin-Nkonga[6], such an approach can be extended to many other physical features. Nevertheless, as far as we are concerned, it still remains a lot of key points to investigate:

- the mathematical analysis of the amplification properties of system $(\mathbf{S})$;

- the validation of numerical schemes for this system and the study of various physical regimes;

- the limits of our asymptotic approach for ultrafast and/or intense pulses by the (numerical) comparison of $(\mathbf{S})$ with the initial Maxwell-Bloch system (M) ;

- the refinement of our model by the introduction of Doppler and Zeeman effects.

\section{Acknowledgment}

This work has been partially supported by the CEA/DAM and by A.C.I Jeunes chercheurs "solutions oscillantes d'EDP", ministère de la Recherche, France. We want to thank D.Benisti for fruitful discussions about the physical model.

\section{References}

[1] C.Besse, B.Bidegaray, Numerical study of self-focusing solutions to the Schrödinger-Deybe system, M2AN, 35, pp. 35-55 (2001).

[2] B.Bidegaray, De Maxwell-Bloch à Schrödinger non-linéaire: une hiérarchie de modèles en optique quantique, HdR (2003). 
[3] B.Bidegaray, A.Bourgeade, D.Reignier, R.Ziolkowski, Multi-level MaxwellBloch simulations, Mathematical and Numerical aspects of Wave propagation (2000).

[4] R.W.Boyd, Nonlinear Optics, Academic Press (1992).

[5] H.Brezis, T.Gallouet, Nonlinear Schroödinger evolution equations, Nonlinear analysis, Theory, Methods $\&$ Applications, Vol. 4, 4, pp. 677-681 (1981).

[6] T.Colin, B.Nkonga, Numerical model for light interaction with two-level atom medium, Université Bordeaux 1, to appear in Physica D (2003).

[7] P.Donnat, Quelques contributions mathématiques en optique non-linéaire, Thèse de doctorat, Ecole Polytechnique (1994).

[8] P.Donnat, J.Rauch, Global solvability of the Maxwell-Bloch equations from nonlinear optics, Arch.Rat.Mech.Anal. 136 (1996).

[9] E.Dumas, Existence globale pour les systèmes de Maxwell-Bloch, preprint Université J.Fourier, Grenoble, exposed at Ecole Polytechnique (2003).

[10] E.Dumas, Diffractive optics with curved phases: beam dispersion and transitions between light and shadow, Asymptotic Analysis (2003).

[11] J.-L.Joly, G.Métivier, J.Rauch, Transparent nonlinear geometric optics and Maxwell-Bloch equations, J.Differential Equations, 166, pp. 175-250 (2000).

[12] J.-L.Joly, G.Métivier, J.Rauch, Global solutions to Maxwell equations in a ferromagnetic medium, Annales Henri Poincaré, 1, no. 2, pp. 307-340 (2000).

[13] D.J.Kaup, A.Reiman, A.Bers, Space-time evolution of nonlinear three-wave interaction, Reviews of Modern Physics, Vol. 51 (April 1979).

[14] D.Lannes, Quelques phénomènes d'interaction d'ondes en optique non linéaire, Thèse de doctorat, Ecole Mathématique et Informatique, Bordeaux 1 (2000).

[15] A.Newell, J.Moloney, Nonlinear Optics (1991). 